\title{
QTL for Main Stem Node Number and Its Response to Plant Densities in 144 Soybean FW-RILs
}

\author{
Wen-Xia $\mathrm{Li}^{1+}$, Ping Wang ${ }^{1,2+}$, Hengxing Zhao ${ }^{1}$, Xu Sun ${ }^{1}$, Tao Yang ${ }^{1}$, Haoran $\mathrm{Li}^{1}$, \\ Yongqin Hou ${ }^{1}$, Cuiqiao Liu $^{1}$, Mahfishan Siyal ${ }^{1}$, Rameez Raja veesar ${ }^{1}$, Bo Hu ${ }^{1 *}$ and \\ Hailong Ning ${ }^{1 *}$ \\ ${ }^{1}$ Key Laboratory of Soybean Biology, Ministry of Education, Key Laboratory of Soybean Biology and Breeding/Genetics, \\ Ministry of Agriculture, Northeast Agricultural University, Harbin, China, ${ }^{2}$ High Education Institute, Huaiyin Institute of \\ Technology, Huai'an, China
}

OPEN ACCESS

Edited by: Yuan-Ming Zhang,

Huazhong Agricultural

University, China

Reviewed by:

Liu Zhangxiong,

Institute of Crop Sciences, Chinese

Academy of Agricultural

Sciences, China

Guizhen Kan,

Naniing Agricultural University, China

*Correspondence:

$\mathrm{Bo} \mathrm{Hu}$

hb554512341@163.com

Hailong Ning

ninghailongneau@126.com

tThese authors have contributed equally to this work

Specialty section:

This article was submitted to

Plant Breeding,

a section of the journal

Frontiers in Plant Science

Received: 11 February 2021

Accepted: 21 July 2021

Published: 20 August 2021

Citation:

Li W-X, Wang P, Zhao H, Sun $X$, Yang T, Li H, Hou Y, Liu C, Siyal M, Raja veesar $R, H u B$ and Ning $H$

(2021) QTL for Main Stem Node Number and lts Response to Plant Densities in 144 Soybean FW-RILs.

Front. Plant Sci. 12:666796.

doi: 10.3389/fpls.2021.666796
Although the main stem node number of soybean [Glycine max (L.) Merr. ] is an important yield-related trait, there have been limited studies on the effect of plant density on the identification of quantitative trait loci (QTL) for main stem node number (MSNN). To address this issue, here, 144 four-way recombinant inbred lines (FW-RILS) derived from Kenfeng 14, Kenfeng 15, Heinong 48, and Kenfeng 19 were used to identify QTL for MSNN with densities of $2.2 \times 10^{5}$ (D1) and $3 \times 10^{5}$ (D2) plants/ha in five environments by linkage and association studies. As a result, the linkage and association studies identified 40 and 28 QTL in D1 and D2, respectively, indicating the difference in QTL in various densities. Among these QTL, five were common in the two densities; 36 were singly identified for response to density; 12 were repeatedly identified by both response to density and phenotype of two densities. Thirty-one were repeatedly detected across various methods, densities, and environments in the linkage and association studies. Among the 24 common QTL in the linkage and association studies, 15 explained a phenotypic variation of more than 10\%. Finally, Glyma.06G094400, Glyma.06G147600, Glyma.19G160800.1, and Glyma.19G161100 were predicted to be associated with MSNN. These findings will help to elucidate the genetic basis of MSNN and improve molecular assistant selection in high-yield soybean breeding.

Keywords: soybean, node number of main stem, response to density, linkage analysis and GWAS, gene mining

\section{INTRODUCTION}

Since soybean was one of the important crops worldwide, it has been an ongoing aim for soybean breeders to breed high yield cultivars in order to meet increasing global demand. As a major plant architecture trait, main stem node number (MSNN) affects soybean seed yield (Yao et al., 2015; Chang et al., 2018), for it is related with seed yield characters, such as logging, number of pods per plant, and days to flowering (Chapman et al., 2003; Zhang et al., 2004; Egli, 2013). MSNN is a typical quantitative trait, and the interaction of the genotype and the environment complicates the study on genetic basis. Therefore, molecular markers are widely used to locate quantitative trait loci (QTL) to reveal the molecular mechanism of MSNN in soybean yield. To date, Soybase (https://www.soybase.org/) has listed 37 QTL for MSNN by genetic linkage analysis (Zhang et al., 2004; Chen et al., 2007; Gai et al., 2007; Li et al., 2010; Liu et al., 2011; Moongkanna et al., 2011; Yao et al., 2015) and 11 quantitative trait nucleotides (QTNs) by genome-wide association study (GWAS) (Fang et al., 2017). 
Genetic linkage analysis is an effective and traditional method to identify genetic intervals that associated plant phenotypes of traits (Tanksley et al., 1992). With the further development of DNA chip technology, single nucleotide polymorphism (SNP) has been widely used in high-density genetic linkage map construction and mapping of QTL (Hyten et al., 2010; Kim et al., 2010; Akond et al., 2013; Jun et al., 2014; Lee et al., 2015). GWAS can identify QTNs in genome regions based on the density of SNP, with advantages of high detection accuracy, high throughput, low cost, and time-saving. However, high false positive ratio is its inevitable defect. More researchers supported the viewpoint that the combination of linkage and association analysis was more accurate and effective than single methods (Ott et al., 2011; Liu et al., 2018; Fang et al., 2020; Zhang Y. C. et al., 2020). However, identifying the location of MSNN QTL for soybean using the combination of linkage analysis and GWAS analysis has not yet been discussed.

Population selection is the most important foundation for the linkage and GWAS analyses to map QTL. Linkage analysis typically is based on populations derived from two parents, and its detection power is usually relatively lower because of its less genetic diversity (Zhang S. et al., 2017). GWAS analysis generally uses natural populations or germplasm resources, and the population structure problem reduces the accuracy of the results. In order to solve the problems, scientists suggested constructing a special population, such as multi-parent advanced generation inter-cross (MAGIC) (Kover et al., 2009). The great opportunity for recombination in multiple parent populations increases mapping accuracy. Abundant genetic variation improves the efficiency of the detection of QTL, and clear kinship in progenies solves the population structure problem (Cavanagh et al., 2008). Kover et al. (2009) first constructed a MAGIC population with 19 Arabidopsis thaliana parents, and proved that the MAGIC population had great advantages in the location of QTL by mapping several known QTL with high precision. Huang et al. (2012) created a wheat MAGIC population from four excellent Australian varieties and identified QTL for plant height and hectoliter weight successfully. Butrón et al. (2019) also identified QTL for resistance to Fusarium ear rot in a MAGIC maize population.

In this study, in order to identify more accurate QTL and further perform gene mining precisely, an FW-RIL derived from a four-way cross was used to identify QTL for the MSNN of two densities in five environments by the combination of linkage analysis and GWAS. This research will enrich MSNN QTL and improve the precision of gene mining, as well as reveal the molecular mechanisms of MSNN in response to density, which will subsequently lay the foundation for marker-assisted selection breeding to increase soybean yield.

\section{MATERIALS AND METHODS}

\section{Plant Materials}

To construct a four-way recombinant inbred line population, four soybean varieties with different node numbers in the main stem, Kenfeng 14, Kenfeng 15, Heinong 48, and Kenfeng 19, were used as parents. In 2008, two single crosses of Kenfeng 14 $\times$ Kenfeng 15 and Heinong $48 \times$ Kenfeng 19 were obtained in Harbin, Heilongjiang province, China, and the $F_{1}$ was crossed as (Kenfeng $14 \times$ Kenfeng 15) $\times$ (Heinong $48 \times$ Kenfeng 19) in 2009. From 2010 to 2014, the progeny was self-crossed following the single seed descent method in Harbin and Yacheng, Hainan province, China. Finally, an FW-RIL population with 144 homozygous individuals was obtained and used for genetic map construction and mapping of QTL.

\section{Field Experiment and Trait Measurement}

The field experiment was conducted in Harbin (E126.63 $\left.\mathrm{N} 45.75^{\circ}\right)$ in $2015(\mathrm{E} 1)$, Keshan $\left(\mathrm{E} 125.64^{\circ}, \mathrm{N} 48.25^{\circ}\right)$ in 2015 (E2), Acheng $\left(\mathrm{E} 127.63^{\circ}, \mathrm{N} 45.82^{\circ}\right)$ in 2016 (E3), Shuangcheng $\left(\mathrm{E} 126.92^{\circ}, \mathrm{E} 45.75^{\circ}\right)$ in 2016 (E4), and Harbin in 2016 (E5). The parents and FW-RILs were planted in a three-row $5 \times$ $0.7 \mathrm{~m}$ plot in a split block design of three replications. The main block arranged the plant densities, namely, $2.2 \times 10^{5}$ plants/ha (D1) and $3 \times 10^{5}$ plants/ha (D2). The sub-blocks were planted lines. The management procedures followed the normal production practices.

Five mature plants of the four parents and 144 four-way recombinant inbred lines (FW-RILs) were selected randomly in the middle of each row to measure MSNN before the harvest in the field for each replication. MSNN indicated the number of nodes from the cotyledonary node to the top of the main stem. The average of the three replications was used for phenotypic data analysis.

\section{Genotyping and SNP Map Construction}

Juvenile leaves were frozen in liquid nitrogen from the parents and FW-RIL plants, and then were ground into powder. Total genomic DNA was extracted with the CTAB method (Doyle et al., 1990) and eluted in 50- $\mu$ l deionized water. SNP genotyping was conducted with SoySNP660K BeadChip at Beijing Boao Biotechnology Co. Ltd. A total of 109,676 SNPs were selected from 600,010 across 20 chromosomes, with minor allele frequency (MAF) $>0.05$ and maximum SNP deletion locus $<20 \%$ as criteria for the screening of SNP quality, and heterozygous loci were marked as missing to better estimate marker effect. Then, the locus was selected at each $100 \mathrm{~kb}$ interval along each chromosome from $3^{\prime}$-bottom to $5^{\prime}$-bottom. 2,292 high-quality SNPs on 20 chromosomes following Mendelian segregating ratio was applied to construct linkage map by the software GAPL V1.0 (Zhang S. et al., 2017). The length of the 20 linkage groups ranged from 76.4 to $329.7 \mathrm{~cm}$, and the total length was $3,539.7 \mathrm{~cm}$. The markers in each linkage group ranged from 16 to 316 , with an average interval distance of $4.09 \mathrm{~cm}$ (ranging from 1.92 to $10.93 \mathrm{~cm}$ ).

\section{Statistical Analysis}

\section{Phenotypic Variation Analysis}

The maximum, minimum, and standard deviations, skewness, and kurtosis of MSNN were calculated for each density in 
each environment. ANOVA was conducted with SAS V 9.2. ANOVA for single environment was carried out according to the following equation:

$$
x_{i j r}=\mu+R_{r}+D_{j}+R D_{j r}+G_{i}+G D_{i j}+\varepsilon_{i j r}
$$

where $x_{i j r}$ is the $r$ th observation of the $i$ th genotype under the $j$ th density in an environment; $\mu$ is the grand mean; $R_{r}$ is the effect of main block $r ; D_{j}$ is the effect of density $j ; R D_{j r}$ is error of main block; $G_{i}$ is the effect of genotype $i ; G D_{i j}$ is the interaction effect of genotype $i$ by density $j$; and $\varepsilon_{i j r}$ is the residual error, $\varepsilon_{i j r} \sim N(0$, $\left.\sigma^{2}\right)$.

For multiple environments, joint ANOVA was conducted according to the following equation:

$$
\begin{aligned}
x_{e i j r} & =\mu+E_{e}+E_{e}\left(R_{r}\right)+D_{j}+E D_{e j}+E_{e}\left(R D_{r j}\right)+G_{i}+G D_{i j} \\
& +G E_{e i}+G D E_{e i j}+\varepsilon_{e i j r}
\end{aligned}
$$

where $x_{e i j r}$ is the $r$ th observation of the $i$ th genotype under the $j$ th density in eth environment; $\mu$ is the grand mean; $E_{e}$ is effect of eth environment; $E_{e}\left(R_{r}\right)$ is the effect of $r$ th main block in $e$ th environment; $D_{j}$ is the effect of density $j ; E D_{e j}$ is the interaction effect of density $j$ by environment $e$; $E_{e}\left(R D_{r j}\right)$ is error of main block in eth environment; $G_{i}$ is the effect of genotype $i$; $G D_{i j}$ is the interaction effect of genotype $i$ by density $j ; G E_{e i}$ is the interaction effect of genotype $i$ by environment $e$; $G D E_{e i j}$ is the interaction effect of genotype $i$ by density $j$ by environment $e$; and $\varepsilon_{\text {eijr }}$ is the residual error, $\varepsilon e_{i j r} \sim N\left(0, \sigma^{2}\right)$.

Genotype variance, genotype $\times$ density interaction variance, and error variance were estimated via a mixed model. The heritability $\left(h^{2}\right)$ for single environment was calculated with the following equation:

$$
h^{2}=\frac{\sigma_{G}^{2}}{\sigma_{G}^{2}+\sigma_{G D}^{2} / d+\sigma^{2} / d r}
$$

The heritability $\left(h^{2}\right)$ for multiple environments was calculated with the following equation:

$$
h^{2}=\frac{\sigma_{G}^{2}}{\sigma_{G}^{2}+\sigma_{G D}^{2} / d+\sigma_{G D E}^{2} / d e+\sigma^{2} / e d r}
$$

where $h^{2}$ is heritability; $\sigma_{G}^{2}$ is the variance of genotype; $\sigma_{G D}^{2}$ is the variance of genotype $\times$ density interaction; $\sigma_{G D E}^{2}$ is the variance of genotype $\times$ density $\times$ environment interaction; $\sigma^{2}$ is the variance of error; $e$ is the number of environments; $d$ is the number of planting density; and $r$ is the number of repetitions.

\section{Response to Density Estimation}

Response to density refers to the difference in node number in response to change in density (from D1 to D2). Response to density (RD) could be evaluated according to the conditional variable method (Zhu, 1995) with the following equation:

$$
R_{D}=x_{D_{2}}-C_{D_{1} D_{2}}\left(x_{D_{1}}-\bar{x}_{D_{1}}\right) / V_{D_{1}}
$$

where $R_{D}$ is the response to density; $x_{D 1}$ is the phenotype value under the density of $\mathrm{D} 1 ; x_{D 2}$ is the phenotype value under the density of $\mathrm{D} 2 ; C_{D 1 D 2}$ is the covariance between phenotypes of MSNN under the two densities; and $\bar{x}_{D 1}$ and $V_{D 1}$ are the average and variance of MSNN under the density of D1, respectively.

\section{Linkage Analysis}

Based on the SNP linkage map constructed above, interval mapping (IM) and inclusive composite interval mapping (ICIM) methods were used to map the QTL for MSNN in every density and environment through the PLQ function of GAPL software V1.0 (Zhang S. et al., 2017). In order to determine the existence of QTL, the scanning step was set to $1 \mathrm{~cm}$, and the likelihood of odds (LOD) threshold was set to 3 . The QTL were named qlNN-chromosome-sequence number or qlRDNN-chromosome-sequence number. The QTL mapped to the same marker region were given the same sequence number. QTL mapping results were mapped on chromosomes with MapChart2.1 (https://www.wur.nl/en). For QTL for MSNN detected in one interval, QTL by density effect in each environment, i.e., the additive effect over two densities, and additive $\times$ density interaction effect, were estimated. The formulas are shown as follows:

$$
\begin{aligned}
\mu_{. .} & =\frac{1}{g d r} \sum_{i, j, k} y_{i j k} \\
\mu_{i j} & =\frac{1}{r} \sum_{i k} y_{i j k} \\
G_{i} & =\frac{1}{d r} \sum_{j, k} y_{i j k}-\mu_{. .} \\
D_{j} & =\frac{1}{g r} \sum_{i, k} y_{i j k}-\mu_{. .} \\
G D_{i j} & =\mu_{i j}-\mu_{. .}-G_{i}-D_{j} \\
\sigma_{G}^{2} & =\sum_{i} f_{i} G_{i}^{2} \\
\sigma_{D}^{2} & =\frac{1}{d} \sum_{j} D_{j}^{2} \\
\sigma_{G D}^{2} & =\sum_{i, j} f_{i j} G D_{i j}^{2} \\
\sigma_{p}^{2} & =\frac{1}{g d r} \sum_{i, j, k}\left(y_{i j k}-\mu_{. .}\right)^{2}
\end{aligned}
$$

where $y_{i j k}$ is the $k$ th phenotype of $i$ th allelic genotype in $j$ th environment, $\mu_{\text {.. }}$ is the grand mean of all observation, $\mu_{\mathrm{ij}}$ is the mean of $i$ th allelic genotype in $j$ th environment, $G_{i}$ is the $i$ th allele effect genotype of putative QTL, $D_{j}$ is the $j$ th density effect, $G D_{i j}$ is the QTL $\times$ density interaction effect of $i$ th allele genotype under $j$ th density, $\sigma_{G}^{2}$ is the genetic variance, $\sigma_{E}^{2}$ is the variance of density effect, $\sigma_{G D}^{2}$ is the variance of the QTL $\times$ density interaction effect, $\sigma_{p}^{2}$ is the phenotypic variance, and $g, d$, and $r$ are the numbers of allelic genotype, density, and replication. On the basis of estimated $\sigma_{G}^{2}, \sigma_{D}^{2}, \sigma_{G D}^{2}$, and $\sigma_{p}^{2}$, the phenotypic variation explanation ratio $(\%)$ of additive $\left(P V E_{A}\right)$ and additive $\times$ density interaction $\left(P V E_{A D}\right)$ effect were estimated 
TABLE 1 | Summarization of phenotype of nod number in main stem.

\begin{tabular}{|c|c|c|c|c|c|c|c|c|c|c|}
\hline \multirow[t]{2}{*}{ Treatments $^{a}$} & \multirow[t]{2}{*}{ Kenfeng 14} & \multirow[t]{2}{*}{ Kenfeng 15} & \multirow[t]{2}{*}{ Heinong 48} & \multirow[t]{2}{*}{ Kenfeng 19} & \multicolumn{4}{|c|}{ FW-RIL } & \multirow[b]{2}{*}{ Skew } & \multirow[b]{2}{*}{ Kurt } \\
\hline & & & & & Min & Max & Mean & Std & & \\
\hline E1D1 & 14.50 & 19.00 & 9.33 & 13.67 & 7.00 & 25.00 & 14.89 & 3.22 & 0.26 & 0.55 \\
\hline E1D2 & 15.00 & 18.50 & 9.70 & 14.20 & 8.00 & 21.00 & 15.74 & 2.81 & -0.43 & -0.51 \\
\hline E2D1 & 13.55 & 16.67 & 10.20 & 13.0 & 8.80 & 17.60 & 11.77 & 1.73 & 0.59 & 0.48 \\
\hline E2D2 & 16.80 & 18.50 & 13.00 & 15.00 & 12.00 & 22.00 & 16.68 & 2.04 & -0.09 & -0.04 \\
\hline E3D1 & 14.33 & 16.50 & 9.33 & 13.67 & 6.67 & 20.00 & 12.58 & 2.73 & 0.38 & -0.38 \\
\hline E3D2 & 13.20 & 17.67 & 8.90 & 12.90 & 5.67 & 19.33 & 12.49 & 2.86 & 0.10 & -0.28 \\
\hline E4D1 & 16.17 & 15.33 & 12.17 & 14.67 & 7.67 & 17.50 & 12.92 & 1.88 & -0.21 & -0.11 \\
\hline E4D2 & 15.50 & 16.33 & 12.67 & 13.33 & 8.00 & 20.00 & 13.45 & 2.50 & 0.09 & -0.18 \\
\hline E5D1 & 15.00 & 17.00 & 10.50 & 14.50 & 7.50 & 22.50 & 14.02 & 2.80 & 0.12 & 0.15 \\
\hline E5D2 & 14.25 & 17.00 & 11.50 & 15.50 & 7.50 & 24.50 & 15.03 & 2.77 & 0.21 & 0.53 \\
\hline
\end{tabular}

${ }^{a}$ E1, Harbin in 2015; E2, Keshan in 2015; E3, Acheng in 2016; E4, Shuangcheng in 2016; E5, Harbin in 2016; D1, normal density (2.2 × $10^{5}$ plants/ha); D2, high density (3 × $10^{5}$ plants/ha).

by the following formula:

$$
\begin{aligned}
P V E_{A} & =\sigma_{G}^{2} \times 100 / \sigma_{p}^{2} \\
P V E_{A D} & =\sigma_{G E}^{2} \times 100 / \sigma_{p}^{2}
\end{aligned}
$$

\section{Genome-Wide Association Studies}

The analysis of population structure was performed with the software STRUCTURE V 2.3.4. The number of subpopulations value $(\mathrm{K})$ was determined with STRUCTURE HARVESTER (http://taylor0.biology.ucla.edu/structureHarvester). Linkage disequilibrium (LD) was analyzed with TASSEL 5.0. The K value was 2 , and the $\mathrm{LD}$ was $1.63 \mathrm{Mb}$. The procedure is described in detail in a previous study (Zhang et al., 2018). Then, the GWAS was conducted with the software mrMLM.GUI V3.0 (Zhang Y. W. et al., 2020). Five multiple locus GWAS methods, mrMLM (Wang et al., 2016), FASTmrMLM (Tamba et al., 2017), FASTmrEMMA (Wen et al., 2018), pLARmEB (Zhang J. et al., 2017), and ISIS EM-BLASSO (Tamba et al., 2017), were used to identify significant QTL that control MSNN and its response to density. The probability $P$ in the first step was set at 0.01 for mrMLM, FASTmrMLM, pLARmEB, ISIS EM-BLASSO, and 0.005 for FASTmrEMMA. The critical LOD score was set at 3 to determine significant QTL. The QTL were named $q n N N$-chromosome-sequence number or qnRDNN-chromosome-sequence number.

\section{Candidate Gene Prediction}

The QTL used to search candidate genes should satisfy the following conditions: (1) for QTL detected by linkage: should be detected in different densities, methods, or environments; explain the phenotypic variation more than $10 \%$, and the interval length should be $<600 \mathrm{~kb}$; (2) for QTL detected by GWAS: should be detected in different densities, with more than two multiple locus GWAS methods, in multiple environments, or by co-location with QTL; and explain the phenotypic variation more than 10\%. The Glyma.Wm82.a2.v1 gene model in Soybase (https://soybase.org/) was used to identify genes at the interval of each of the QTL (at the interval of $100 \mathrm{~kb}$ on either side, determined by the rate of LD decay). According to the Phytozome website (https://phytozome.jgi.doe.gov), genes highly expressed in the stem or shoot tip were selected among them. Then, the selected genes were put together to conduct pathway analysis on the Kyoto Encyclopedia of Genes and Genomes (KEGG) website (http://www.kegg.jp). Finally, the candidate genes were predicted through the results of pathway analysis combined with their homologous genes information on other crops and potential functions in GO number (https://www.ebi.ac.uk/QuickGO/) and the NCBI database (http://www.ncbi.nlm.nih.gov/).

\section{RESULTS AND ANALYSIS}

\section{Phenotypic Analysis}

The summary of MSNN phenotype is presented in Table $\mathbf{1}$. The data showed that the node number of the parents and FW-RIL varied with density and environment. The range of FW-RIL covered the parents, which indicated strong bilateral transgressive segregation. The skewness and kurtosis values of the FW-RIL ranged from -1 to 1 , and the phenotypic data displayed a typical normal distribution (Figure 1). All the characters of phenotypic variation indicated that MSNN was controlled by large- and small-effect QTL. The significant genotypic and genotype $x$ environment interaction variance indicated a substantial genetic variation of MSNN existed among the FW-RILs and response of genotypes to environment varied among different environments. On the basis of the significant genotype $\times$ density interaction variance and genotype $x$ density $x$ environment interaction variance, it was implied that the MSNN response differed to the densities and that the response varied among the environments (Table 2).

Comparing the two densities, the mean of MSNN in D2 was higher than that in D1 (Figure 2), indicating the existence of MSNN response to density. The difference in heritability among 


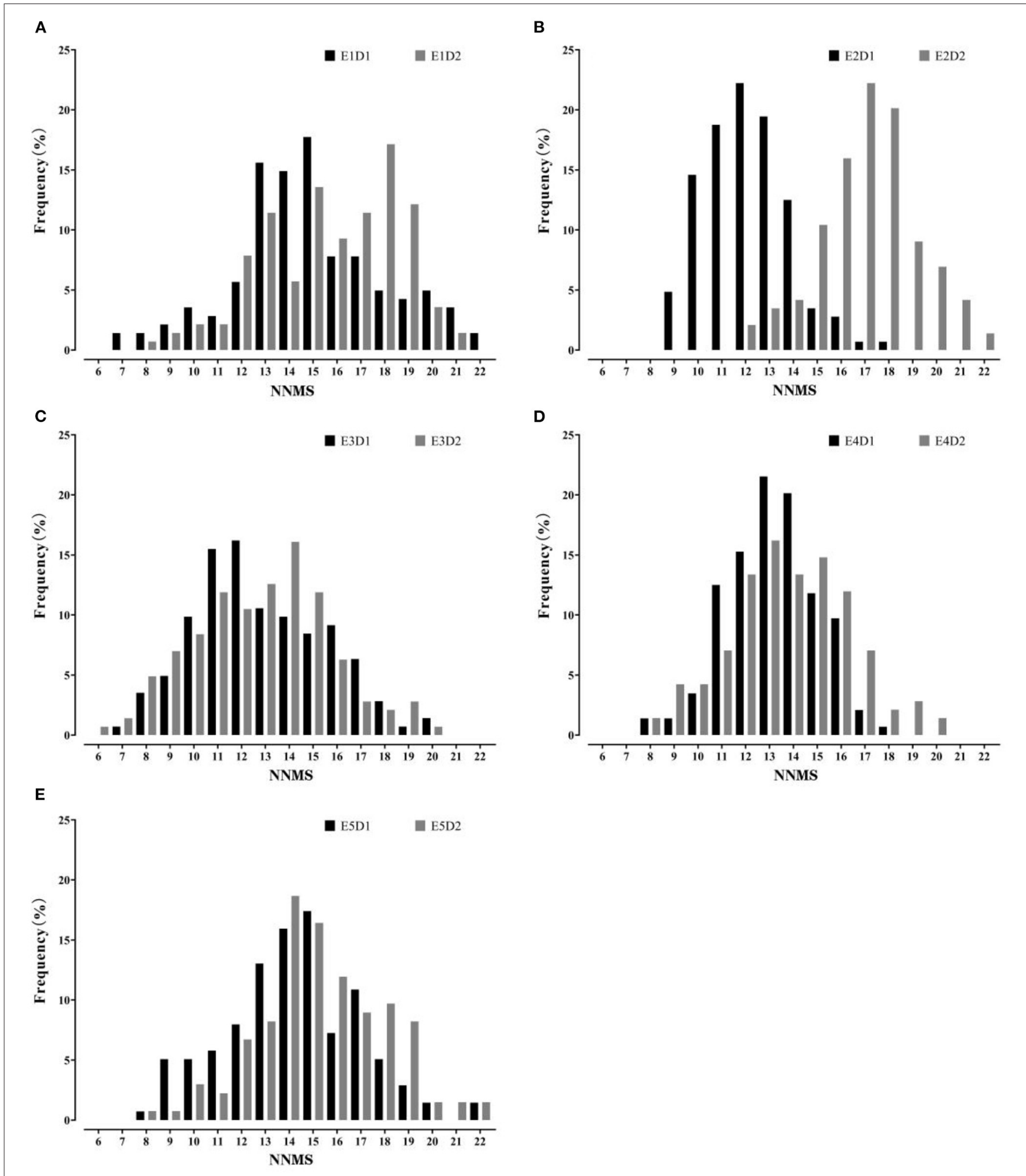

FIGURE 1 | Frequency distribution of main stem node number (MSNN) in the four-way recombinant inbred line (FW-RIL) population in different treatments. (A-E) show the distribution in E1, E2, E3, E4, and E5, respectively; E1, Harbin in 2015; E2, Keshan in 2015; E3, Acheng in 2016; E4, Shuangcheng in 2016; E5, Harbin in 2016; D1, normal density $\left(2.2 \times 10^{5}\right.$ plants/ha); D2, high density $\left(3 \times 10^{5}\right.$ plants/ha). 
TABLE 2 | Variance and heritability of nod number in main stem of four-way recombinant inbred lines.

\begin{tabular}{|c|c|c|c|c|c|c|c|c|c|}
\hline \multirow[t]{2}{*}{ Environment ${ }^{a}$} & \multicolumn{2}{|c|}{ Genotype } & \multicolumn{2}{|c|}{ Genotype $\times$ Density } & \multicolumn{2}{|c|}{ Genotype $\times$ Environment } & \multicolumn{2}{|c|}{ Genotype $\times$ Density x Environment } & \multirow[t]{2}{*}{$h^{2}$} \\
\hline & MS & Variance & MS & Variance & MS & Variance & MS & Variance & \\
\hline Joint & $69.79^{\star \star}$ & 1.75 & $16.13^{\star \star}$ & 0.05 & $18.36^{\star \star}$ & 0.35 & $15.97^{\star \star}$ & 4.37 & 0.74 \\
\hline E1 & $31.83^{\star \star}$ & 0.38 & $30.00^{\star \star}$ & 8.96 & & & & & 0.07 \\
\hline E2 & $17.97^{\star \star}$ & 1.47 & $9.13^{\star \star}$ & 2.05 & & & & & 0.49 \\
\hline E3 & $32.34^{\star \star}$ & 2.37 & $18.32^{\star \star}$ & 5.04 & & & & & 0.44 \\
\hline E4 & $24.72^{\star \star}$ & 2.32 & $10.89^{\star \star}$ & 2.71 & & & & & 0.56 \\
\hline E5 & $35.81^{\star \star}$ & 4.16 & $11.92^{\star \star}$ & 3.04 & & & & & 0.68 \\
\hline
\end{tabular}

${ }^{a} E 1$, Harbin in 2015; E2, Keshan in 2015; E3, Acheng in 2016; E4, Shuangcheng in 2016; E5, Harbin in 2016.

** Significant difference at level of $P<0.01$.

the environments suggested the genetic basis for the formation of MSNN change according to environment. The extreme difference in heritability between joint and single environments showed that the response of genotype to change in density varied among environments. The whole variation in density under environments showed it was possible to detect different QTL in various environments.

\section{Mapping of QTL for MSNN}

In this study, 38 QTL for MSNN were detected on 18 chromosomes (except chromosome 7 and 15) with LOD value of over 3, which explained $3.44-14.93 \%$ of phenotypic variance (Figure 3, Supplementary Table 1). Among these QTL, 13 were identified in D1, seven in D2, and five in both D1 and D2. For 14 QTL underlying the response of MSNN to density, three and three were associated singly with MSNN in D1 and D2, and one of which was associated simultaneously with MSNN in D1 and D2 (Figure 4).

In the five different environments, eight, three, three, six, and 12 quantitative trait loci (QTL) were detected in E1, E2, E3, E4, and E5, respectively. Six QTL could be found in more than two environments: qlNN-6-2 in E3 and E4; qlNN-8-2 in E3 and E5; qlNN-9-2 (qlRDNN-9-2) in E1, E2, and E3; qlNN10-2 ( $q l R D N N-10-1)$ in E2 and E3; qlNN-13-1 ( $q l R D N N-13-$ 1 ) in E4 and E5; and qlNN-17-1 (qlRDNN-17-1) in E1 and E5, respectively (Figure 5). Besides, 13 QTL could be detected with both the IM and ICIM methods, and in two densities and more than two environments, they could be considered as stable (Table 3). Among them, 12 QTL explained the phenotypic variation over $10 \%$. qlNN-1-3 was detected in E2D1 with PVE of $11.01 \%$; qlRDNN-3-1 was detected in E2RD with PVE of 7.5712.07\%; qlNN-6-1 (qlRDNN-6-1) was detected in E1D1, E1D2, and E1RD with PVE of 5.4-12.5\%; qlNN-6-2 was detected in E3D1, E4D1, and E4D2 with PVE of 6.85-11.68\%; qlNN-8-2 was detected in E3D1, E5D1, and E5D2 with PVE of 6.8211.56\%; qlNN-9-2 (qlRDNN-9-2) was detected in E1D2, E2D2, E2RD, and E3D2 with PVE of 6.63-10.50\%; qlNN-9-3 was detected in E4D1 and E4D2 with PVE of 5.81-11.75\%; qlNN-102 ( $q$ lRDNN-10-1) was detected in E2RD and E3D1with PVE of 6.12-11.02\%; qlNN-13-1 (qlRDNN-13-1) was detected in E4RD and E5D2 with PVE of 5.16-10.07\%; qlNN-17-1 (qlRDNN-171) was detected in E1D1 and E5RD with PVE of 3.44-12.96\%;
qlNN-18-1 was detected in E5D1 and E5D2 with PVE of 6.72$10.02 \%$; and qlNN-19-1 was detected in E4D1 with PVE of 10.73$14.93 \%$.

Of all the QTL, the genome length of 15 QTL was $<600 \mathrm{~kb}$, which included six of the 13 ones: qlRDNN-3-1, qlRDNN-32, qlNN-6-1 (qlRDNN-6-1), qlNN-6-2, qlNN-17-1 (qlNN-17-1), and $q l N N-19-1$. The other stable QTL were repeatedly identified at a wide interval. In addition, all of the stable QTL with genome length of $<600 \mathrm{~kb}$ could explain the phenotypic variation more than $10 \%$ except qlRDNN-3-2. Consequently, these intervals might play a critical role in mining genes to regulate MSNN.

Among all the alleles from the 13 stable QTL for MSNN, the parent Kenfeng 14 carried the positive additive effect alleles for 7 QTL, Kenfeng 15 for 11 QTL, Heinong 48 for 7 QTL, and Kenfeng 19 for 8 QTL. Four, four, one and one QTL from Kenfeng14, Kenfeng 15, Heinong 48, and Kenfeng 19 could obviously increase MSNN (additive effect > 1). Oppositely, Kenfeng 14 carried the negative additive effect alleles for 0 QTL, Kenfeng 15 for 6 QTL, and Heinong 48 and Kenfeng 19 for 10 QTL. Three, two, seven and four QTL from Kenfeng 14, Kenfeng 15, Heinong 48, and Kenfeng 19 could obviously decrease MSNN (additive effect $<-1$ ) (Table 3).

In the five environments, the total of $\mathrm{PVE}_{\mathrm{A}}$ and $\mathrm{PVE}_{\mathrm{AE}}$ varied extremely, ranging from 1.97 (in E2) to $45.98 \%$ (in E5). It was shown that the genetic basis of MSNN response to density varied in different environments (Table 4).

By comparison of $\mathrm{PVE}_{\mathrm{A}}$ and PVE $\mathrm{AD}, 15$ QTL (qlNN-20-1, qlNN-12-1, qlNN-3-1, qlNN-3-2, qlNN-13-1, qlNN-19-1, qlNN8-2, qlNN-8-1, qlNN-6-4, qlNN-17-2, qlNN-9-3, qlNN-18-1, qlNN-9-1, qlNN-18-2, and qlNN-12-2) expressed stably in two densities. Of these, qlNN-3-2 in E1, qlNN-9-2 and qlNN-14-1 in E4, and qlNN-3-1, qlNN-6-4, qlNN-8-1, qlNN-8-2, qlNN9-1, qlNN-12-1, qlNN-12-2, qlNN-13-1, and qlNN-18-1 in E5 showed consistency with $\mathrm{PVE}_{\mathrm{A}}$ over $2 \%$.

Twelve QTL (qlNN-8-3, qlNN-2-1, qlNN-11-1, qlNN-14-1, qlNN-1-2, qlNN-17-1, qlNN-1-3, qlNN-10-2, qlNN-6-3, qlNN10-1, qlNN-18-3, and qlNN-1-1) showed larger inconformity in various densities. Among these, qlNN-1-2 and qlNN-11-1 in E1, and qlNN-10-2 in E3 were expressed differently in specific density with PVE $\mathrm{AD}$ more than 2\%. Three QTL (qlNN-5-1, qlNN-9-2, qlNN-6-2) responded differently to density change in various environments. 

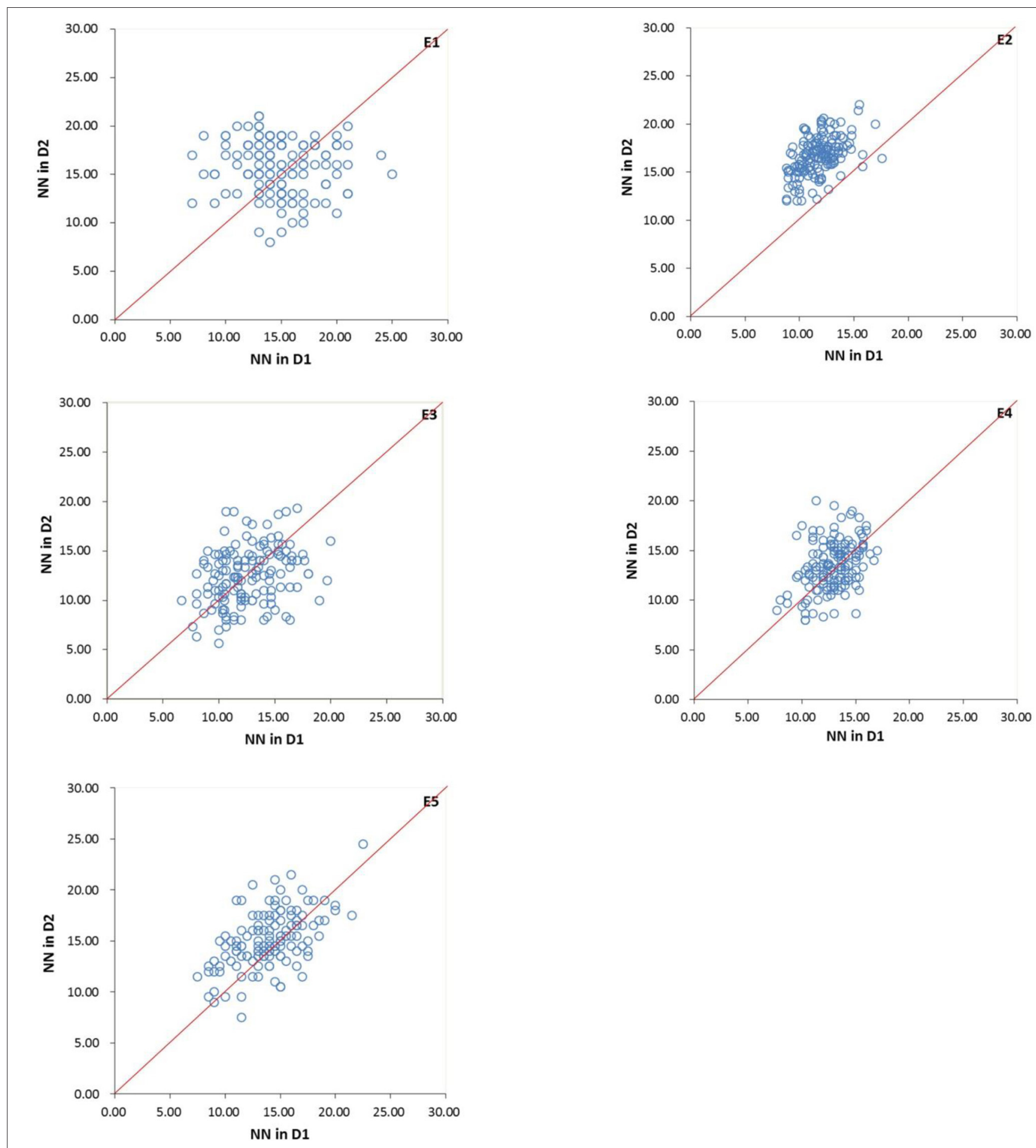

FIGURE 2 | Variation in main stem node number (MSNN) under two densities in five environments for four-way recombinant inbred line (FW-RIL). E1, Harbin in 2015; E2, Keshan in 2015; E3, Acheng in 2016; E4 Shuangcheng in 2016; E5, Harbin in 2016; D1, normal density (2.2 × $10^{5}$ plants/ha); D2, high density (3 $\times 10^{5}$ plants/ha).

\section{QTL by GWAS}

By GWAS analysis, QTL associated with MSNN were detected all over the genome on 18 chromosomes except chromosomes 16 and 17 (Figure 3). Thirty-four QTL were found in D1,
18 in $\mathrm{D} 2$, and 34 in $\mathrm{RD}$, in which five were simultaneously found in D2 and RD (Figure 4). In other words, a total of 81 QTL were found, 47 of which could explain 10.1$38.38 \%$ phenotypic variation (Supplementary Table 2). From 


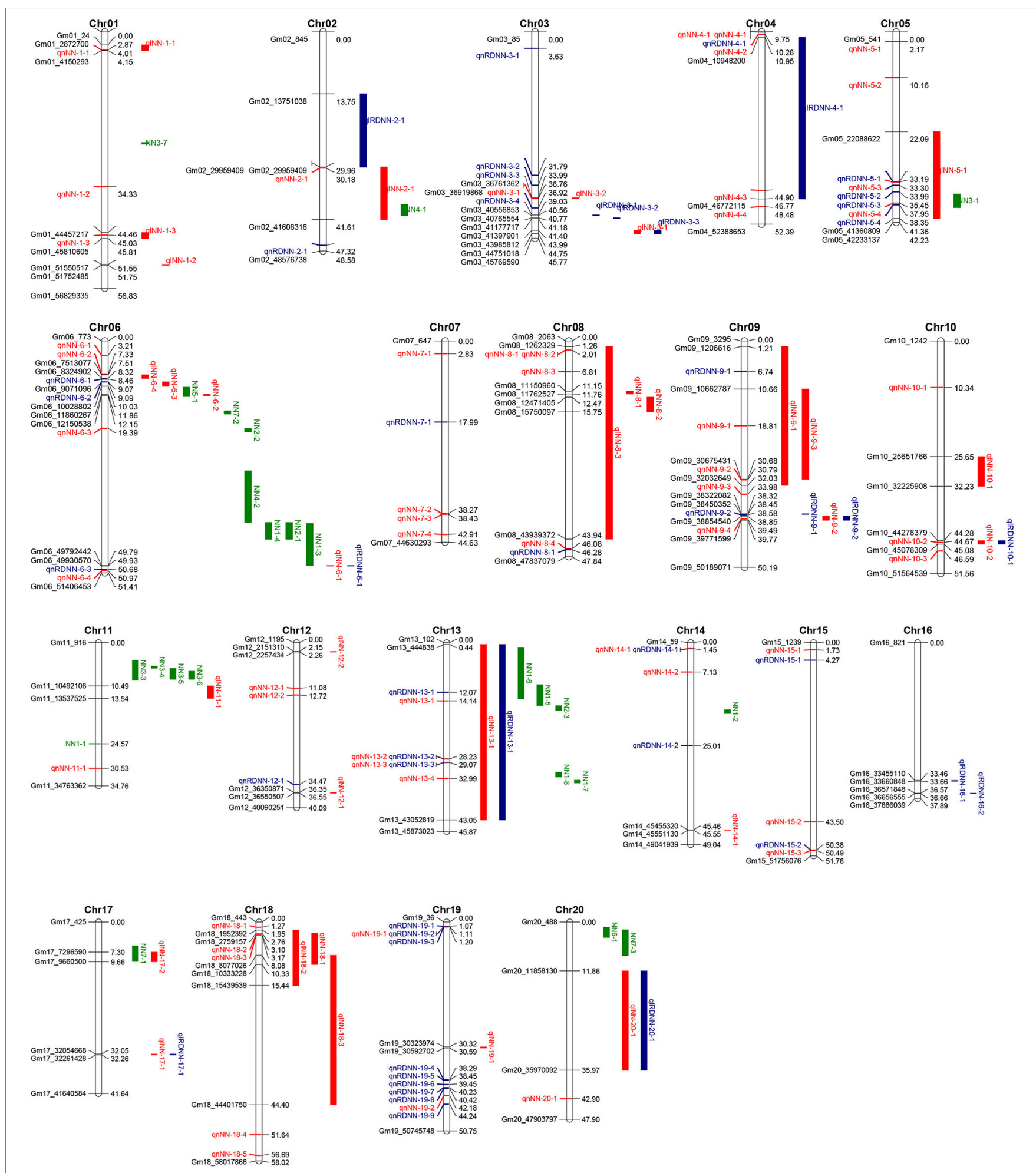

FIGURE 3 | Distribution of quantitative trait loci (QTL) underlying main stem node number (MSNN) on 20 chromosomes. The red and blue colors represent QTL controlling NN and response to density change identified in present research, respectively, and the green color represents QTL underlying NN identified in previous research listed in Soybase (www.soybase.org). 

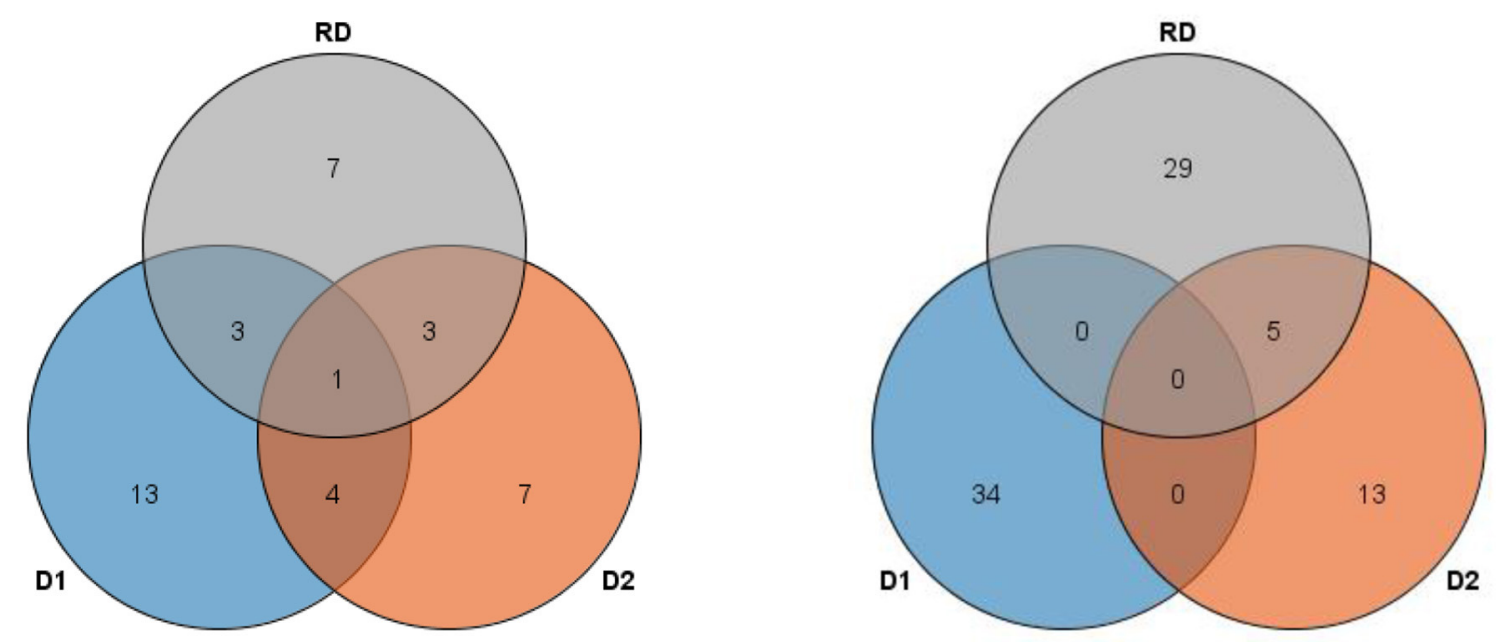

FIGURE 4 | Venn figure for frequency of linkage (left) and genome-wide association study (GWAS) (right) quantitative trail loci (QTL) underlying main stem node number (MSNN) and response to density increment. D1, normal density ( $2.2 \times 10^{5}$ plants/ha); D2, high density (3 $\times 10^{5}$ plants/ha); RD, response to density.

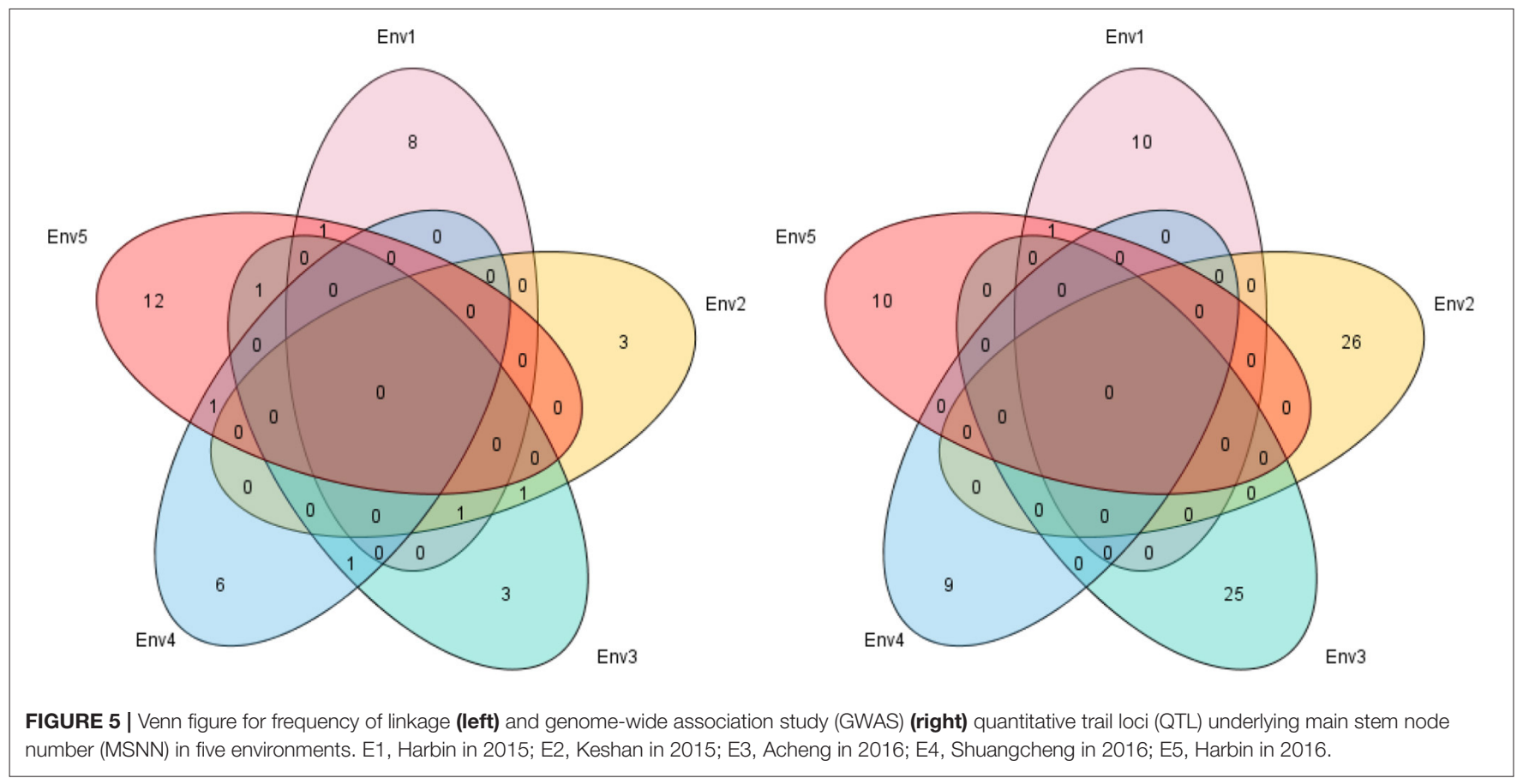

the different environments, 10, 26, 25, 9, and 10 QTL were identified specifically in E1, E2, E3, E4, and E5, respectively, and one QTL ( $q n R D N N-13-3)$ was repeatedly identified in E1 and E5 (Figure 5). Twenty-four stable QTL could be found with multiple methods or in the environments, 19 of which could explain phenotypic variation more than $10 \%$ (Table 5). $q n N N-$ 4-1 (qnRDNN-4-1) was identified in E4D2 and E4RD with PVE of 11.684-28.71\%; qnNN-4-2 was identified in E5D1 with PVE of 15.1315-15.3806\%; qnRDNN-5-3 was identified in E2RD with PVE of 8.3101-18.7664\%; $q n N N-6-2$ was identified in E4D2 with PVE of 10.7297-17.4975\%; qnRDNN-7-1 was identified in
E5RD with PVE of 9.9725-24.8721\%; qnNN-7-2 was identified in E2D1 with the PVE of 20.9359-26.3968\%; qnNN-7-4 was identified in E2D2 with PVE of 12.1529-13.9883\%; qnNN-9-1 was identified in E2D1 with PVE of 5.1691-10.674\%; qnRDNN9-2 was identified in E3RD with PVE of 11.9235-13.3229\%; $q n N N-10-2$ was identified in E4D1 with PVE of 9.192510.6283\%; qnNN-11-1 was identified in E5D1 with PVE of 26.5631-26.5932\%; qnNN-12-1 was identified in E1D1 with PVE of 15.6919-22.8492\%; qnRDNN-13-1 was identified in E2RD with PVE of 10.5682-20.7672\%; qnNN-13-2 ( $q n R D N N-13-2)$ was identified in E3D2 and E3RD with PVE of 4.6444-16.7223\%; 
TABLE 3 | Stable quantitative trait loci (QTL) for main stem node number (MSNN) identified under different densities in different environments with different methods.

\begin{tabular}{|c|c|c|c|c|c|c|c|c|c|c|c|}
\hline QTL & Chromosome & $\begin{array}{l}\text { Physical position of markers } \\
\text { (bp) }\end{array}$ & $\begin{array}{l}\text { Length of markers } \\
\text { (bp) }\end{array}$ & Treatment $^{\mathrm{a}}$ & Method & $\begin{array}{l}\text { LOD } \\
\text { score }\end{array}$ & $\begin{array}{l}\text { PVE }^{\mathrm{b}} \\
(\%)\end{array}$ & Add $^{c}$ & Add2 & Add3 & Add4 \\
\hline \multirow[t]{2}{*}{ qINN-1-3 } & Chr01 & $44,457,217 \ldots 45,810,605$ & $1,353,388$ & E2D1 & ICIM & 3.19 & 11.01 & -0.02 & 0.73 & 0.24 & -0.94 \\
\hline & & & & E2D1 & IM & 3.19 & 11.01 & -0.02 & 0.73 & 0.24 & -0.94 \\
\hline \multirow[t]{2}{*}{ qIRDNN-3-1 } & ChrO3 & $40,556,853 \ldots 40,765,554$ & 208,701 & E2RD & IM & 3.57 & 7.57 & -0.51 & 0.57 & 0.57 & -0.63 \\
\hline & & & & E2RD & ICIM & 4.60 & 12.07 & -0.50 & 0.52 & 0.64 & -0.66 \\
\hline \multirow[t]{2}{*}{ qIRDNN-3-2 } & Chr03 & $41,177,717 \ldots 41,397,901$ & 220,184 & $\mathrm{E} 5 \mathrm{RD}$ & ICIM & 3.81 & 9.55 & -0.52 & 1.16 & -0.95 & 0.31 \\
\hline & & & & E5RD & IM & 3.30 & 3.58 & -0.45 & 1.17 & -1.01 & 0.29 \\
\hline qINN-6-1 & Chr06 & $49,792,442 \ldots .49,930,570$ & 138,128 & E1D2 & ICIM & 3.28 & 12.50 & 1.52 & -1.19 & -0.56 & 0.23 \\
\hline \multirow[t]{5}{*}{ (qIRDNN-6-1) } & & & & E1D1 & ICIM & 4.87 & 5.74 & 1.72 & -0.41 & -1.41 & 0.10 \\
\hline & & & & E1D2 & IM & 3.41 & 11.72 & 1.69 & -1.20 & -0.62 & 0.12 \\
\hline & & & & E1RD & ICIM & 3.41 & 11.11 & 1.59 & -1.22 & -0.49 & 0.12 \\
\hline & & & & E1RD & IM & 3.16 & 11.11 & 1.70 & -1.10 & -0.67 & 0.08 \\
\hline & & & & E1D1 & IM & 3.37 & 5.40 & 2.56 & -0.77 & -1.42 & -0.37 \\
\hline \multirow[t]{6}{*}{ qINN-6-2 } & Chr06 & $11,860,267 \ldots 12,150,538$ & 290,271 & E4D2 & ICIM & 3.70 & 11.68 & 0.79 & 0.67 & -0.08 & -1.37 \\
\hline & & & & E4D2 & $\mathrm{IM}$ & 3.70 & 8.82 & 0.79 & 0.67 & -0.08 & -1.37 \\
\hline & & & & E3D1 & ICIM & 3.35 & 6.85 & 0.65 & 0.93 & 0.06 & -1.64 \\
\hline & & & & E4D1 & ICIM & 4.04 & 8.55 & -0.23 & 0.77 & 0.41 & -0.96 \\
\hline & & & & E3D1 & IM & 3.35 & 6.85 & 0.65 & 0.93 & 0.06 & -1.64 \\
\hline & & & & E4D2 & IM & 3.80 & 9.13 & 0.31 & 0.86 & 0.50 & -1.67 \\
\hline \multirow[t]{5}{*}{ qINN-8-2 } & Chr08 & $12,471,405 \ldots 15,750,097$ & $3,278,692$ & E5D2 & ICIM & 4.40 & 7.14 & 1.37 & 0.60 & -0.73 & -1.24 \\
\hline & & & & E5D1 & ICIM & 5.49 & 10.92 & 0.96 & 0.98 & -1.39 & -0.55 \\
\hline & & & & E5D1 & $\mathrm{IM}$ & 4.72 & 11.56 & 0.88 & 1.23 & -1.44 & -0.67 \\
\hline & & & & E3D1 & ICIM & 3.08 & 6.82 & 0.13 & 1.49 & -0.52 & -1.10 \\
\hline & & & & E3D1 & $\mathrm{IM}$ & 3.08 & 6.82 & 0.13 & 1.49 & -0.52 & -1.10 \\
\hline qINN-9-2 & Chr09 & $38,854,540 \ldots 39,771,599$ & 917,059 & E1D2 & ICIM & 3.02 & 10.50 & -1.09 & -0.09 & -0.02 & 1.19 \\
\hline \multirow[t]{3}{*}{ (qIRDNN-9-2) } & & & & E3D2 & ICIM & 3.07 & 7.94 & -0.91 & 0.97 & -0.86 & 0.81 \\
\hline & & & & E2D2 & IM & 3.61 & 9.91 & -0.79 & 1.06 & 0.07 & -0.34 \\
\hline & & & & E2RD & $\mathrm{IM}$ & 3.17 & 6.63 & -0.76 & 0.84 & 0.12 & -0.20 \\
\hline \multirow[t]{2}{*}{ qINN-9-3 } & Chr09 & $10,662,787 \ldots 30,675,431$ & $20,012,644$ & E4D1 & IM & 3.29 & 5.81 & 0.28 & -0.04 & 0.64 & -0.88 \\
\hline & & & & E4D2 & ICIM & 3.73 & 11.75 & -1.92 & 0.49 & 1.03 & 0.39 \\
\hline$q I N N-10-2$ & Chr10 & $44,278,379 \ldots 45,076,309$ & 797,930 & E3D1 & ICIM & 3.02 & 6.12 & -0.06 & 0.80 & -1.31 & 0.56 \\
\hline \multirow[t]{3}{*}{$(q I R D N N-10-1)$} & & & & E3D1 & IM & 3.02 & 6.12 & -0.06 & 0.80 & -1.31 & 0.56 \\
\hline & & & & E2RD & IM & 3.37 & 8.41 & -1.05 & -0.16 & 0.56 & 0.65 \\
\hline & & & & E2RD & ICIM & 3.72 & 11.02 & -0.75 & -0.43 & 0.59 & 0.60 \\
\hline qINN-13-1 & Chr13 & $444,838 \ldots 43,052,819$ & $42,607,981$ & E4RD & ICIM & 3.26 & 10.07 & 2.29 & -0.62 & -0.90 & -0.77 \\
\hline \multirow[t]{3}{*}{ (qIRDNN-13-1) } & & & & E4RD & IM & 3.26 & 10.07 & 2.29 & -0.62 & -0.90 & -0.77 \\
\hline & & & & E5D2 & ICIM & 3.35 & 5.16 & 1.34 & 0.25 & 0.33 & -1.92 \\
\hline & & & & E5D2 & $\mathrm{IM}$ & 3.05 & 8.31 & 1.81 & 0.07 & 0.05 & -1.93 \\
\hline$q I N N-17-1$ & Chr17 & $32,054,668 \ldots 32,261,428$ & 206,760 & E1D1 & ICIM & 9.51 & 12.96 & 0.82 & 1.95 & -1.20 & -1.57 \\
\hline
\end{tabular}




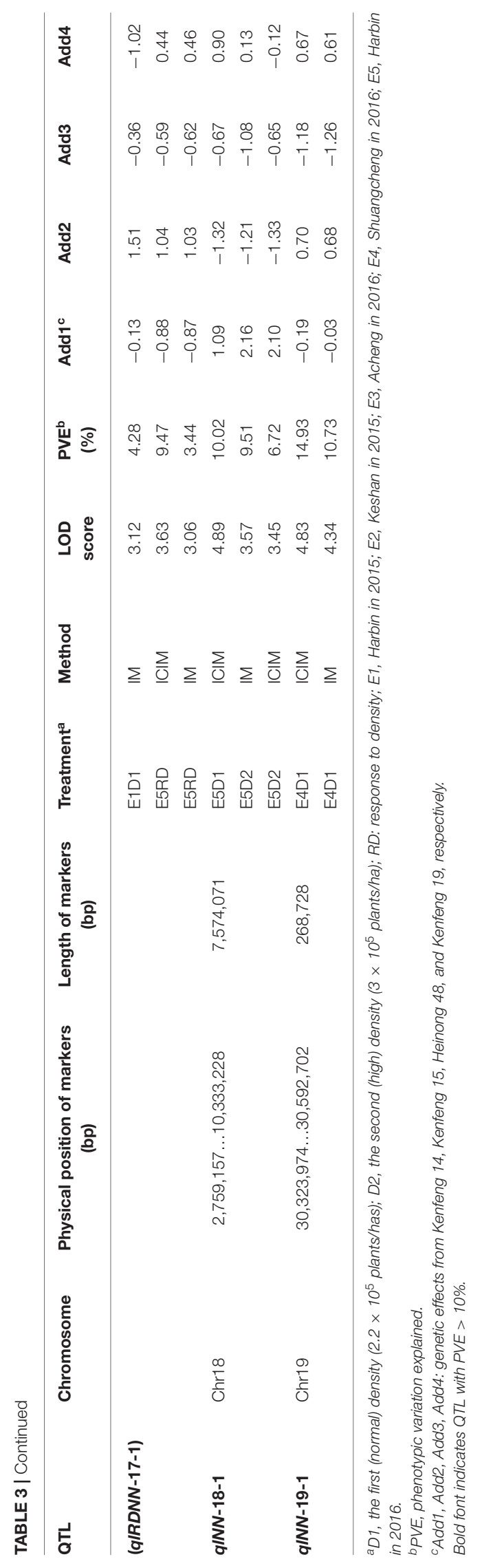

qnNN-13-3 (qnRDNN-13-3) was identified in E1D2, E1RD, and E5RD with PVE of 14.1841-18.659\%; qnNN-14-1 (qnRDNN14-1) was identified in E1D2 and E1RD with PVE of 24.064538.3834\%; qnNN-15-1 was identified in E4D1 with PVE of 13.4194-24.9554\%; $q n N N-18-3$ was identified in E2D1 with PVE of 11.7346-20.1023\%; and $q n N N-19-2$ was identified in E2D1 and could explain phenotypic variation of 4.3512-10.302\%.

Among the whole QTL identified by GWAS, 24 were colocated in the interval of QTL detected by linkage analysis (Figure 3), 15 of which could explain phenotypic variation more than 10\%: qnRDNN-5-3 in qlNN-5-1, qnRDNN-9-2 in qlRDNN9-1, qnNN-10-2 (qlRDNN-10-1) in qlNN-10-2, qnRDNN-131, $q n N N-13-2$ ( $q n R D N N-13-2)$ and $q n N N-13-3$ ( $q n R D N N-13-$ 3) in $q l N N-13-1$ ( $q l R D N N-13-1), q n N N-18-3$ in $q l N N-18-1$, $q n N N-1-1$ in $q l N N-1-1, q n N N-1-3$ in $q l N N-1-3$, qnNN-4-3 in qlRDNN-4-1, qnRDNN-5-1 in qlNN-5-1, qnRDNN-9-1 in qlNN9-1, qnNN-9-4 in qlNN-9-2 (qlRDNN-9-2), qnNN-13-4 in qlNN13-1 ( $q$ lRDNN-13-1), and $q n N N-18-2$ in $q l N N-18-1$. These QTL also could be considered stable because of the detection by linkage analysis and GWAS.

\section{Candidate Gene Prediction}

In this study, genes were screened based on the physical position of the five stable QTL (genome length $<600 \mathrm{~kb}$ and PVE $>10 \%$ ) and the 27 stable one (PVE $>10 \%$ ) mentioned above. In total, 549 genes were found, among which 265 were highly expressed in the stem or shoot tip. Then these genes were used to conduct pathway analysis in the KEGG database (http://www.kegg.jp).

A total of 106 genes (which accounted for 40\%) were annotated and divided into 36 catalogs and three protein families (Figure 6). Among these genes, four (Glyma.06G094400, Glyma.06G147600, Glyma.19G160800, and Glyma.19G161100) were speculated as potential candidate genes to regulate MSNN (Table 6).

\section{DISCUSSION}

\section{Superiority of Using FW-RIL Population}

In this study, a FW-RIL population was used for mapping, a simple mode of MAGIC (Kover et al., 2009) with four parents. It kept the advantage of the MAGIC population in abundant genetic variation. When mapping QTL, it could be applied to analyze allelic additive effects value from four parents. As long as there were differences between any two parents, QTL could be detected. For example, qlNN-1-1, qlNN8-2, and qlNN-11-1 could not be detected in a bi-parent population derived from Kenfeng $14 \times$ Kenfeng 15, because the allelic additive effects from the parents were approximately equivalent (Supplementary Table 1); so, the more allelic additive effect differences in the FW-RIL population, the greater the improvement made in QTL detection. Moreover, FW-RIL was an artificial population without the population structure problem, which was suitable for GWAS analysis.

Although the population size was relatively small, the combination of the linkage and GWAS analyses could improve mapping power. Furthermore, the experiment was conducted in three environments, which could compensate for the shortage in 
TABLE 4 | Additive and additive by density effect of quantitative trait loci (QTL) under two densities in each environment.

Environment ${ }^{\mathrm{a}}$ QTL

Additive effect ${ }^{b}$

Additive by density effect

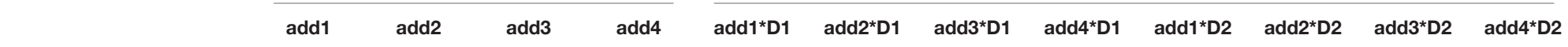

E1

\begin{tabular}{|c|c|c|c|c|c|c|c|c|c|c|c|c|c|c|}
\hline qINN-1-2 & -0.50 & 0.55 & -0.06 & 0.02 & -0.48 & 0.80 & -0.31 & 0.00 & 0.48 & -0.80 & 0.31 & 0.00 & 1.72 & 3.25 \\
\hline qINN-2-1 & 0.15 & 0.16 & -0.30 & -0.01 & 0.41 & -0.01 & -0.23 & -0.16 & -0.41 & 0.01 & 0.23 & 0.16 & 0.49 & 0.54 \\
\hline qINN-3-2 & -0.32 & 0.64 & -0.48 & 0.15 & -0.25 & 0.43 & 0.00 & -0.18 & 0.25 & -0.43 & 0.00 & 0.18 & 2.27 & 0.82 \\
\hline qINN-5-1 & 0.17 & -0.13 & 0.16 & -0.20 & -0.52 & 0.35 & -0.17 & 0.34 & 0.52 & -0.35 & 0.17 & -0.34 & 0.28 & 1.18 \\
\hline qINN-9-2 & -0.44 & 0.28 & 0.53 & -0.37 & -0.26 & -0.16 & 0.68 & -0.26 & 0.26 & 0.16 & -0.68 & 0.26 & 1.76 & 1.51 \\
\hline qINN-10-1 & 0.04 & 0.18 & 0.08 & -0.30 & 0.28 & 0.19 & -0.51 & 0.03 & -0.28 & -0.19 & 0.51 & -0.03 & 0.36 & 1.06 \\
\hline qINN-11-1 & 0.08 & 0.13 & 0.10 & -0.31 & 0.92 & -0.08 & -0.54 & -0.30 & -0.92 & 0.08 & 0.54 & 0.30 & 0.30 & 2.96 \\
\hline qINN-17-1 & 0.13 & 0.25 & -0.18 & -0.21 & 0.48 & 0.27 & -0.28 & -0.47 & -0.48 & -0.27 & 0.28 & 0.47 & 0.47 & 1.68 \\
\hline qINN-17-2 & -0.04 & 0.45 & 0.17 & -0.58 & -0.01 & -0.06 & 0.19 & -0.13 & 0.01 & 0.06 & -0.19 & 0.13 & 1.61 & 0.14 \\
\hline qINN-18-2 & 0.23 & -0.08 & -0.27 & 0.13 & 0.15 & 0.09 & -0.16 & -0.08 & -0.15 & -0.09 & 0.16 & 0.08 & 0.42 & 0.17 \\
\hline Total & & & & & & & & & & & & & 15.15 & 16.91 \\
\hline qINN-9-2 & 0.07 & 0.24 & -0.19 & -0.12 & -0.13 & -0.11 & 0.13 & 0.11 & 0.13 & 0.11 & -0.13 & -0.11 & 0.33 & 0.15 \\
\hline qINN-1-3 & -0.01 & 0.23 & -0.16 & -0.06 & -0.11 & 0.31 & 0.10 & -0.31 & 0.11 & -0.31 & -0.10 & 0.31 & 0.27 & 0.54 \\
\hline qINN-20-1 & -0.13 & 0.29 & -0.21 & 0.06 & 0.13 & 0.13 & 0.01 & -0.27 & -0.13 & -0.13 & -0.01 & 0.27 & 0.40 & 0.29 \\
\hline Total & & & & & & & & & & & & & 1.00 & 0.97 \\
\hline qINN-6-2 & -0.43 & 0.13 & -0.34 & 0.64 & 0.01 & 0.09 & 0.29 & -0.38 & -0.01 & -0.09 & -0.29 & 0.38 & 1.94 & 0.66 \\
\hline qINN-8-2 & 0.42 & -0.51 & -0.20 & 0.29 & 0.05 & -0.02 & -0.02 & 0.00 & -0.05 & 0.02 & 0.02 & 0.00 & 1.63 & 0.01 \\
\hline qINN-8-3 & -0.22 & -0.06 & 0.14 & 0.14 & 0.19 & -0.17 & -0.27 & 0.24 & -0.19 & 0.17 & 0.27 & -0.24 & 0.24 & 0.69 \\
\hline qINN-9-2 & -0.28 & 0.12 & 0.08 & 0.08 & 0.22 & -0.14 & 0.19 & -0.27 & -0.22 & 0.14 & -0.19 & 0.27 & 0.27 & 0.59 \\
\hline qINN-10-2 & 0.50 & -0.44 & 0.03 & -0.08 & -0.38 & 0.42 & -0.63 & 0.59 & 0.38 & -0.42 & 0.63 & -0.59 & 1.47 & 3.47 \\
\hline Total & & & & & & & & & & & & & 5.55 & 5.41 \\
\hline qINN-5-1 & 0.26 & -0.30 & -0.26 & 0.30 & -0.16 & 0.16 & 0.06 & -0.05 & 0.16 & -0.16 & -0.06 & 0.05 & 1.65 & 0.25 \\
\hline qINN-6-2 & 0.12 & -0.15 & 0.07 & -0.03 & -0.12 & 0.25 & -0.44 & 0.31 & 0.12 & -0.25 & 0.44 & -0.31 & 0.22 & 1.93 \\
\hline qINN-6-3 & 0.25 & 0.10 & -0.10 & -0.24 & 0.11 & -0.12 & 0.25 & -0.24 & -0.11 & 0.12 & -0.25 & 0.24 & 0.67 & 0.78 \\
\hline qINN-9-2 & 0.35 & -0.08 & 0.19 & -0.46 & -0.09 & 0.08 & 0.29 & -0.28 & 0.09 & -0.08 & -0.29 & 0.28 & 2.27 & 0.98 \\
\hline qINN-9-3 & -0.53 & 0.30 & 0.08 & 0.15 & 0.24 & -0.18 & -0.02 & -0.04 & -0.24 & 0.18 & 0.02 & 0.04 & 1.39 & 0.35 \\
\hline qINN-14-1 & 0.57 & 0.11 & -0.60 & -0.08 & -0.20 & 0.25 & 0.00 & -0.06 & 0.20 & -0.25 & 0.00 & 0.06 & 3.96 & 0.41 \\
\hline qINN-18-3 & -0.27 & 0.12 & -0.16 & 0.32 & 0.11 & -0.19 & 0.48 & -0.41 & -0.11 & 0.19 & -0.48 & 0.41 & 1.09 & 1.98 \\
\hline qINN-19-1 & -0.01 & 0.27 & -0.05 & -0.21 & 0.04 & -0.11 & 0.22 & -0.15 & -0.04 & 0.11 & -0.22 & 0.15 & 0.77 & 0.44 \\
\hline Total & & & & & & & & & & & & & 14.478 & 9.195 \\
\hline qINN-1-1 & 0.03 & -0.39 & 0.19 & 0.18 & -0.29 & 0.30 & 0.33 & -0.34 & 0.29 & -0.30 & -0.33 & 0.34 & 0.82 & 1.27 \\
\hline qINN-3-1 & -1.22 & 0.06 & 0.46 & 0.70 & 0.20 & -0.44 & 0.17 & 0.07 & -0.20 & 0.44 & -0.17 & -0.07 & 4.41 & 0.63 \\
\hline qINN-6-4 & -0.55 & 0.14 & -0.08 & 0.48 & -0.24 & -0.24 & 0.15 & 0.32 & 0.24 & 0.24 & -0.15 & -0.32 & 2.02 & 0.84 \\
\hline qINN-8-1 & -0.52 & -0.66 & 0.61 & 0.57 & 0.05 & 0.29 & -0.25 & -0.09 & -0.05 & -0.29 & 0.25 & 0.09 & 4.39 & 0.44 \\
\hline qINN-8-2 & 0.16 & -0.15 & -0.89 & 0.88 & 0.39 & 0.08 & -0.10 & -0.37 & -0.39 & -0.08 & 0.10 & 0.37 & 4.65 & 0.80 \\
\hline
\end{tabular}




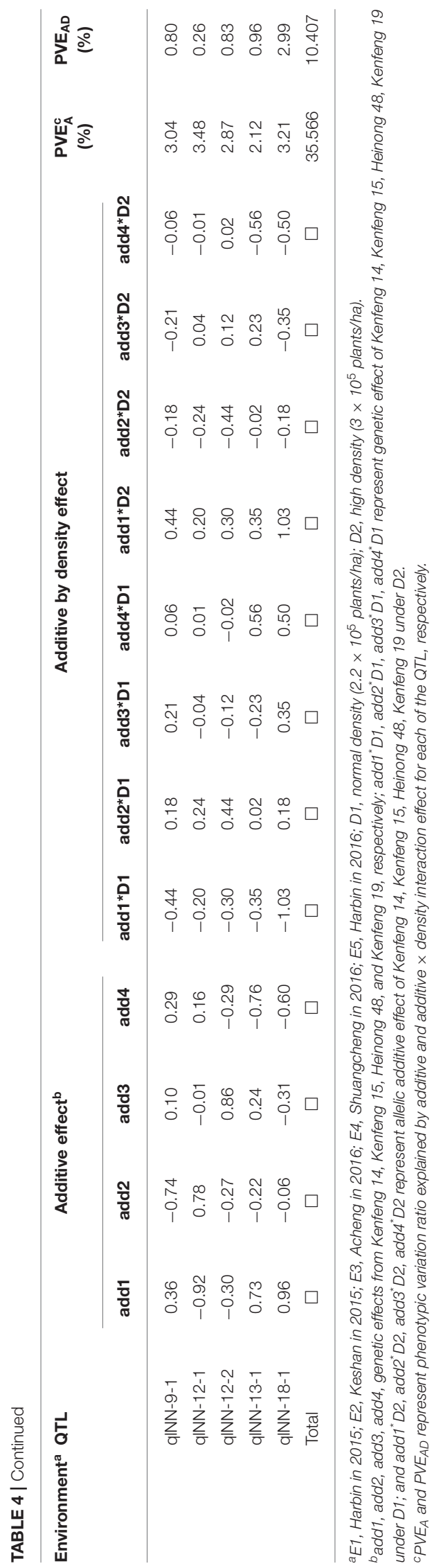

lower power in single environments. In summary, the statistical methods and multiple environment design could increase QTL detection power.

\section{Combination of Genetic Linkage Analysis and GWAS Analysis}

Both the genetic linkage and GWAS analyses were the main methods to identify genome regions related to quantitative traits. As mentioned above, research studies have already combined the two methods to conduct target trait location analysis. In soybean, the combination of the two methods was used in several traits, such as seed size and shape (Hu et al., 2013), seed protein and oil content (Zhang et al., 2019), number of pods (Song et al., 2020), and plant height (Fang et al., 2020). Similar to the MAGIC population, four parents carried multiple allelic genotypes in FW-RIL, so it could conduct linkage and association analysis (Zhang et al., 2018; Li et al., 2019, 2020, 2021; Liu et al., 2019; Qi et al., 2020; Song et al., 2020; Tian et al., 2020; Wang et al., 2021). First, the principles of linkage and association were different: the former associated an interval (region) with a target trait, and the latter associated a position (SNP) with a target trait. Second, the genotype data used in linkage and association analysis were different: the former was based on a small number of markers, and the latter depended on the large amount of markers over the whole genome, so the combination of linkage and GWAS could increase the identification of genomic regions associated with target traits in FW-RIL. In this research, the IM and inclusive composite interval mapping methods were used in the linkage analysis, and mrMLM, FASTmrMLM, FASTmrEMMA, pLARmEB, and ISIS EM-BLASSO were used in the GWASs. A total of 38 QTL were identified by linkage analysis and 81 QTL were identified by GWAS, and 24 QTL were co-located in the interval of the identified QTL. The results indicated that the difference of the two methods in statistical principles and the genetic basis could complement each other and facilitate the detection of QTL.

\section{Density Response of MSNN}

Plant density is considered to be an important factor affecting soybean yield and yield components, such as MSNN. Ikeda et al. (1994) reported that soybean yield increased as density increased because of increase in total node number, especially branch node number. In this study, the MSNN of most of the lines increased as the density increased. Some lines showed the opposite response to density increase, indicating that the expression of gene for MSNN was probably affected by the change in density. By combining linkage and GWAS analyses, 55 QTL were identified in D1, and 33 QTL were identified in D2, respectively. Only five of them were identified in both of the densities, while the rest were detected in the single density. The results showed that the genetic basis of the QTL for MSNN was significantly different in the two densities, and for the genotype, environments, densities and their interaction were all at work. Inspired by the conditional genetic effects (Zhu, 1995) based on a net-effect analysis, the effect of MSNN response to density was estimated by the removal of other factors except planting density increment. In total, 48 QTL for MSNN response to density were identified when planting 
TABLE 5 | Genome-wide association studies for main stem node number (MSNN) detected by multiple methods under different densities in different environments.

\begin{tabular}{|c|c|c|c|c|c|c|c|c|}
\hline QTL & Marker & Chromosome & $\begin{array}{c}\text { Marker } \\
\text { position (bp) }\end{array}$ & Treatment $^{\mathrm{a}}$ & Method & Effect & $\begin{array}{l}\text { LOD } \\
\text { score }\end{array}$ & $r^{2}(\%)^{b}$ \\
\hline \multirow[t]{2}{*}{$\begin{array}{l}q n N N-4-1 \\
\text { (qnRDNN-4-1) }\end{array}$} & AX-157404156 & Chr04 & $9,753,769$ & E4D2 & $\begin{array}{l}\text { ISIS EM-BLASSO, } \\
\text { FASTmrMLM }\end{array}$ & $\begin{array}{l}1.45 \\
1.27\end{array}$ & $\begin{array}{l}5.83 \\
5.83\end{array}$ & $\begin{array}{l}28.71 \\
21.55\end{array}$ \\
\hline & & & & $\mathrm{E} 4 \mathrm{RD}$ & $\begin{array}{l}\text { ISIS EM-BLASSO, } \\
\text { mrMLM }\end{array}$ & $\begin{array}{l}0.90 \\
1.28\end{array}$ & $\begin{array}{l}3.12 \\
3.43\end{array}$ & $\begin{array}{l}11.68 \\
21.65\end{array}$ \\
\hline$q n N N-4-2$ & AX-157124243 & Chr04 & $10,279,678$ & E5D1 & $\begin{array}{l}\text { FASTmrMLM, } \\
\text { pLARmEB }\end{array}$ & $\begin{array}{l}1.14 \\
1.13\end{array}$ & $\begin{array}{l}3.07 \\
3.31\end{array}$ & $\begin{array}{l}15.38 \\
15.13\end{array}$ \\
\hline$q n N N-5-3$ & AX-157344915 & Chr05 & $33,299,938$ & E3D1 & $\begin{array}{l}\text { FASTmrMLM, } \\
\text { pLARmEB }\end{array}$ & $\begin{array}{l}-0.87 \\
-0.90\end{array}$ & $\begin{array}{l}4.17 \\
3.39\end{array}$ & $\begin{array}{l}8.76 \\
9.25\end{array}$ \\
\hline qnRDNN-5-3 & $A X-157510718$ & Chr05 & $35,447,656$ & E2RD & $\begin{array}{l}\text { PLARmEB, } \\
\text { FASTmrMLM }\end{array}$ & $\begin{array}{l}-0.84 \\
-0.53\end{array}$ & $\begin{array}{l}7.67 \\
4.35\end{array}$ & $\begin{array}{r}18.77 \\
8.31\end{array}$ \\
\hline$q n N N-5-4$ & AX-157217038 & Chr05 & $37,951,491$ & E3D2 & $\begin{array}{l}\text { FASTmrMLM, } \\
\text { pLARmEB }\end{array}$ & $\begin{array}{l}-0.78 \\
-0.78\end{array}$ & $\begin{array}{l}4.26 \\
4.26\end{array}$ & $\begin{array}{l}9.10 \\
9.10\end{array}$ \\
\hline$q n N N-6-2$ & AX-117468788 & Chr06 & $7,332,663$ & E4D2 & $\begin{array}{l}\text { ISIS EM-BLASSO, } \\
\text { FASTmrMLM }\end{array}$ & $\begin{array}{l}0.89 \\
1.14\end{array}$ & $\begin{array}{l}3.12 \\
3.12\end{array}$ & $\begin{array}{l}10.73 \\
17.50\end{array}$ \\
\hline$q n R D N N-7-1$ & AX-157553491 & ChrO7 & $17,986,150$ & E5RD & $\begin{array}{l}\text { ISIS EM-BLASSO, } \\
\text { pLARmEB, } \\
\text { FASTmrMLM }\end{array}$ & $\begin{array}{l}-0.97 \\
-0.65 \\
-0.73\end{array}$ & $\begin{array}{l}5.71 \\
3.13 \\
5.33\end{array}$ & $\begin{array}{c}24.87 \\
9.97 \\
13.88\end{array}$ \\
\hline$q n N N-7-2$ & $A X-157107526$ & Chr07 & $38,273,310$ & E2D1 & $\begin{array}{l}\text { ISIS EM-BLASSO, } \\
\text { FASTmrEMMA, } \\
\text { pLARmEB, } \\
\text { mrMLM, } \\
\text { FASTmrMLM }\end{array}$ & $\begin{array}{l}-0.97 \\
-1.84 \\
-0.89 \\
-0.86 \\
-0.95\end{array}$ & $\begin{array}{l}6.49 \\
6.33 \\
7.98 \\
7.02 \\
10.67\end{array}$ & $\begin{array}{l}26.40 \\
22.15 \\
22.41 \\
20.94 \\
25.17\end{array}$ \\
\hline$q n N N-7-4$ & AX-157299646 & Chr07 & $42,911,447$ & E2D2 & $\begin{array}{l}\text { pLARmEB, } \\
\text { FASTmrMLM }\end{array}$ & $\begin{array}{l}0.69 \\
0.64\end{array}$ & $\begin{array}{l}5.97 \\
3.25\end{array}$ & $\begin{array}{l}13.99 \\
12.15\end{array}$ \\
\hline qnNN-8-4 & AX-157333638 & Chr08 & $46,075,240$ & E3D1 & pLARmEB, & $\begin{array}{l}-0.87 \\
-0.71\end{array}$ & $\begin{array}{l}3.30 \\
3.04\end{array}$ & $\begin{array}{l}9.49 \\
6.33\end{array}$ \\
\hline$q n N N-9-1$ & AX-157536173 & Chr09 & $18,807,588$ & E2D1 & $\begin{array}{l}\text { pLARmEB, } \\
\text { FASTmrMLM } \\
\text { FASTmrMLM }\end{array}$ & $\begin{array}{l}-0.44 \\
-0.64 \\
-0.52\end{array}$ & $\begin{array}{l}3.15 \\
5.26 \\
4.93\end{array}$ & $\begin{array}{c}5.17 \\
10.67 \\
7.01\end{array}$ \\
\hline qnRDNN-9-2 & AX-157088086 & Chro9 & $38,577,050$ & E3RD & ISIS EM-BLASSO, & $\begin{array}{l}-0.93 \\
-2.03\end{array}$ & $\begin{array}{l}5.03 \\
3.65\end{array}$ & $\begin{array}{l}11.92 \\
13.32\end{array}$ \\
\hline$q n N N-10-2$ & AX-157499787 & Chr10 & $44,669,350$ & E4D1 & $\begin{array}{l}\text { ISIS EM-BLASSO, } \\
\text { FASTmrEMMA } \\
\text { pLARmEB }\end{array}$ & $\begin{array}{l}0.71 \\
0.69\end{array}$ & $\begin{array}{l}3.37 \\
3.29\end{array}$ & $\begin{array}{r}10.63 \\
9.19\end{array}$ \\
\hline$q n N N-11-1$ & AX-157134381 & Chr11 & $30,526,558$ & E5D1 & $\begin{array}{l}\text { pLARmEB, } \\
\text { FASTmrMLM }\end{array}$ & $\begin{array}{l}1.51 \\
1.51\end{array}$ & $\begin{array}{l}5.76 \\
5.71\end{array}$ & $\begin{array}{l}26.56 \\
26.59\end{array}$ \\
\hline$q n N N-12-1$ & $A X-157131535$ & Chr12 & $11,077,832$ & E1D1 & $\begin{array}{l}\text { PLARmEB, } \\
\text { FASTmrMLM }\end{array}$ & $\begin{array}{l}-1.12 \\
-1.27\end{array}$ & $\begin{array}{l}5.48 \\
3.99\end{array}$ & $\begin{array}{l}15.69 \\
22.85\end{array}$ \\
\hline qnRDNN-13-1 & AX-157183655 & Chr13 & $12,074,020$ & E2RD & $\begin{array}{l}\text { mrMLM, } \\
\text { FASTmrMLM }\end{array}$ & $\begin{array}{l}0.92 \\
0.62\end{array}$ & $\begin{array}{l}3.64 \\
4.80\end{array}$ & $\begin{array}{l}20.77 \\
10.57\end{array}$ \\
\hline
\end{tabular}


TABLE 5 | Continued

QTL

Marker

Chromosome

Marker

Marker
position (bp)

Treatment ${ }^{\mathrm{a}}$

Method

Effect

$28,227,088$

E3D2

ISIS EM-BLASSO,

1.18

LOD

PLARmEB,

(qnRDNN-13-2)

AX-157244239

Chr13

E3D2

pLARmEB

qnNN-13-3

AX-157484481

Chr13

$29,074,011$

E3RD

FASTmrMLM

1.18
0.89
0.89

score

(qnRDNN-13-3)

AX-117471784

qnNN-14-1
(qnRDNN-14-1)

AX-117471784

Chr 14

E1D2

FASTMIMLM,

FASTmrMLM,

ISIS EM-BLASSO

ISIS EM-BLASSO

ISIS EM-BLASSO

mrMLM

ISIS EM-BLASSO,

pLARmEB mrMLM

FASTMrMLM

FASTmrEMMA

PLARMEB,

FASTmrMLM

pLARmEB,

FASTmrMLM

pLARmEB,

FASTMrMLM

pLARmEB,

mrMLM,

FASTmrMLM

aE1, Harbin in 2015; E2, Keshan in 2015; E3, Acheng in 2016; E4, Shuangcheng in 2016; E5, Harbin in 2016; D1, hormal density (2.2 × $10^{5}$ plants/ha); D2, high density (3 × 105 plants/ha).

${ }^{b} r^{2}$, proportion of total phenotypic variation explained by each QTL. Bold font indicates QTL with PVE $>10 \%$. 

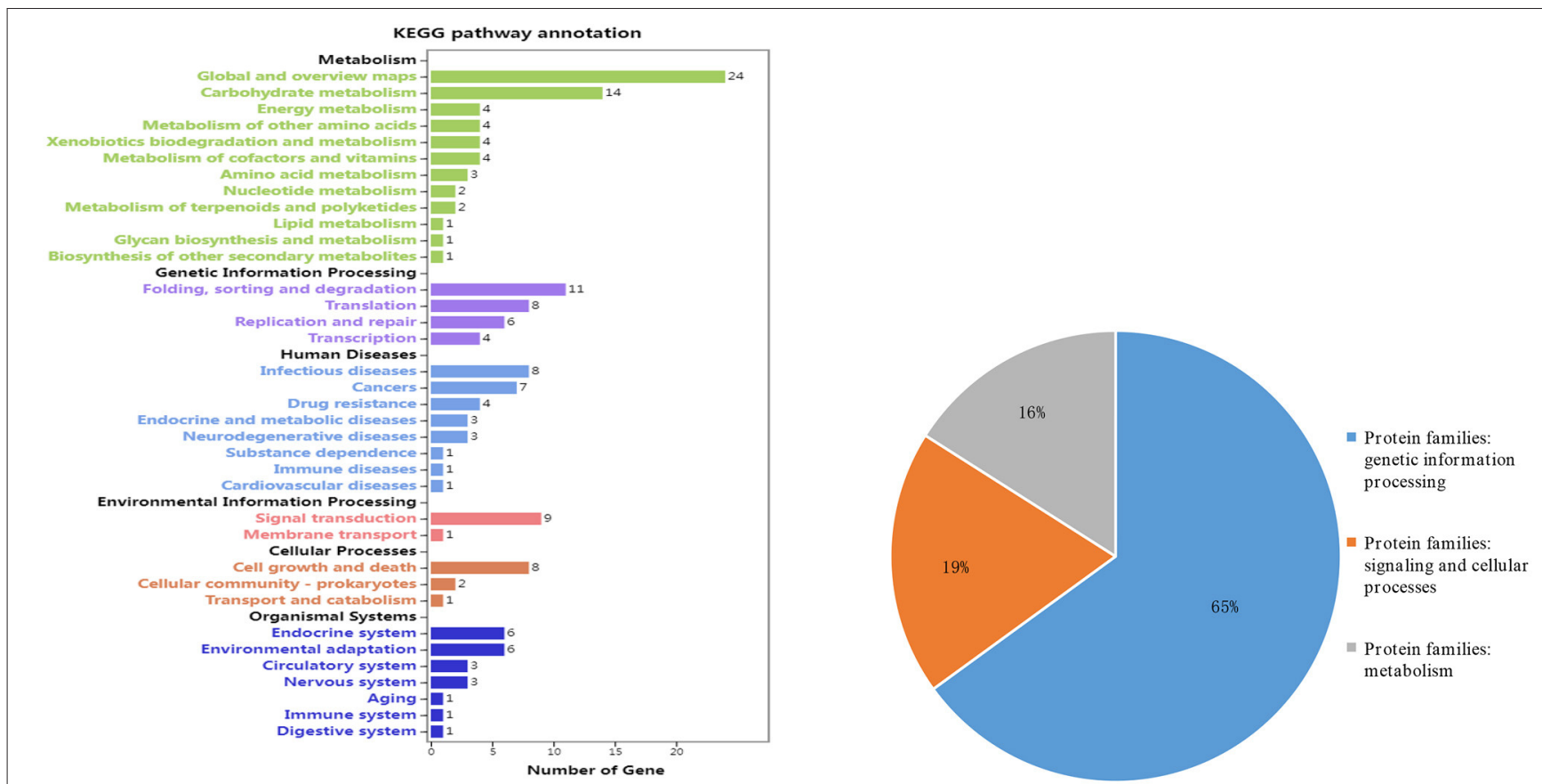

FIGURE 6 | Information on pathways (left) and orthologous protein families (right) of main stem node number (MSNN)-annotated candidate genes.

density increased from $2.2 \times 10^{5}$ (D1) to $3 \times 10^{5}$ plants/ha (D2), which is more valuable for molecular assistance selection (MAS) on MSNN in a specific planting density. Besides, in terms of the additive effects of all QTL for MSNN response to density, Heinong 48 and Kenfeng 14 were relatively suitable parents for increasing and decreasing MSNN in the MAS of soybean breeding, respectively.

\section{Comparison of QTL Identified in Various Genetic Backgrounds}

There were 25 QTL identified by linkage and 11 identified by GWAS listed in Soybase (https://www.soybase.org/search/qtllist_ by_symbol.php). Among the 119 QTL identified this study, 10 had genome intervals that overlapped with published QTL node number (Figure 3). qlNN-2-1 was identified on chromosome 2 in genome intervals of 29,959,409-41,608,316 bp, overlapping with Node number 4-1 (38,221,027-40,699,300 bp) (Liu et al., 2011). qlNN-5-1was identified on chromosome 5 in genome intervals of 22,088,622-41,360,809 bp, overlapping with Node number 31 (35,971,621-38,939,759 bp) (Chen et al., 2007). qlNN-6-2 was identified on chromosome 6 in genome intervals of $11,860,267-$ $12,150,538$ bp, overlapping with Node number 5-1 (10,251,126$12,336,492$ bp) (Moongkanna et al., 2011). qlNN-13-1 (qlRDNN13-1) was identified on chromosome 13 in genome intervals of 444,838-43,052,819 bp, overlapping with Node numbers 15, 1-6, 1-7, 1-8 (Gai et al., 2007), and 2-3 (Zhang et al., 2004). qlNN-17-2 was identified on chromosome 17 in genome intervals of 7,296,590-9,660,500 bp, overlapping with Node number 7-1 (5,788,551-9,576,644 bp) (Li et al., 2010). qnNN5-4 (37,951,491 bp) and qnRDNN-5-4 (38,349,709 bp) were identified in chromosome 5 and fell in the interval of Node number 3-1 (35,971,621-38,939,759 bp) (Chen et al., 2007); qnNN-6-3 (19,386,897 bp) was identified on chromosome 6 and fell in the interval of Node number 2-2 (19,370,872-20,218,893 bp) (Zhang et al., 2004). qnRDNN-13-1 (12,074,020 bp) and qnNN-13-1 (14,139,382 bp) were identified on chromosome 13 and fell in the interval of Node number 1-5 (10,199,530$15,306,234$ bp) (Gai et al., 2007). The rest of 33 QTL identified by linkage and 76 QTL identified by GWAS were newly discovered, among which 37 with PVE $>10 \%$ were repeatedly identified with multiple density, environments, or methods (Supplementary Tables 1, 2). Consequently, this study probably would provide a great number of available genome regions and some potential high-confident candidate genes for MSNN.

\section{Candidate Gene Related With MSNN}

It is known that only few genes were directly related to MSNN in different crops. A novel ricMT gene was highly expressed in stem nodes (Yu et al., 1998). ZmMADS3 was expressed in the stem nodes of maize, and the transgenic maize reduced the number of nodes (Heuer et al., 2001). Dt1 controlled the number of nodes in soybean by regulating stem growth habit (Bernard, 1972). Therefore, it is of great significance to explore potential candidate genes for MSNN. In this study, four among 106 genes were predicted for MSNN.

Brassinosteroids are essential plant hormones with significant effect on cell proliferation and elongation. Glyma.06G147600 was annotated as protein brassinosteroid insensitive 1 (BRI1). It has been demonstrated that BRI1 is a receptor kinase that transduces steroid signals across the plasma membrane, which 


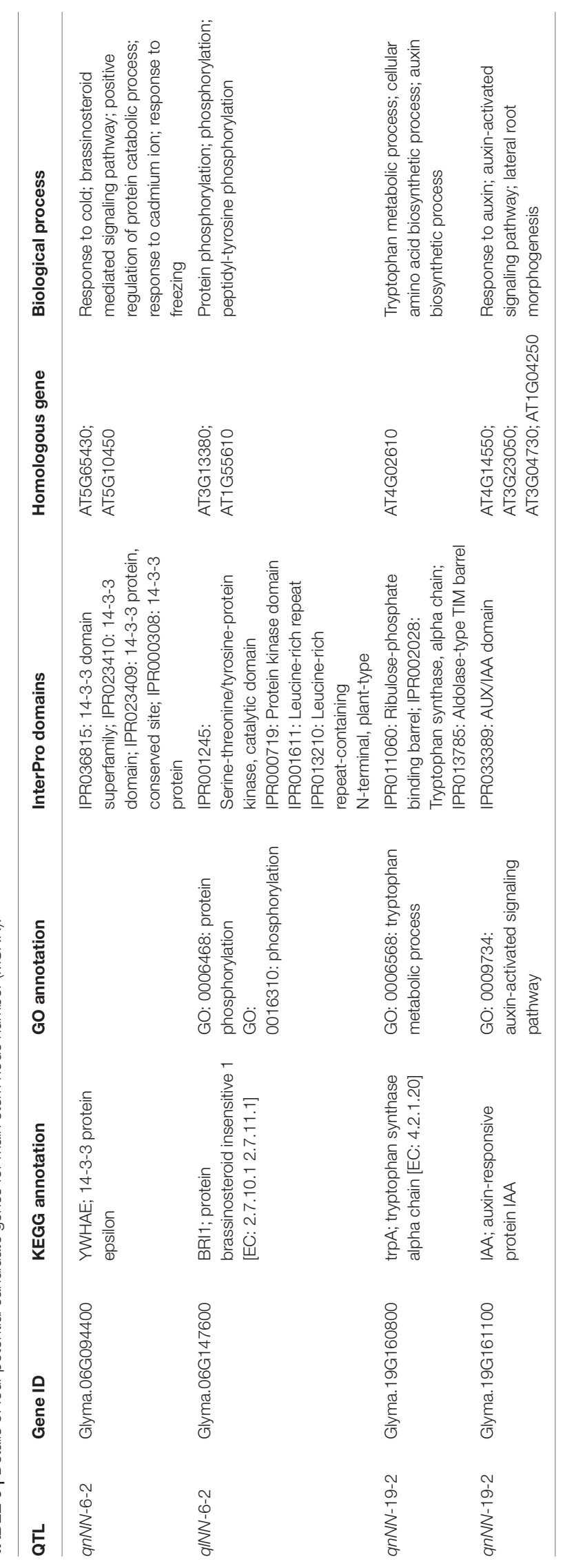

is likely to be the primary brassinosteroids (BR) receptor in Arabidopsis (Wang et al., 2001). Glyma.06G094400 was annotated as 14-3-3 protein epsilon. 14-3-3 proteins were highly conserved regulatory proteins, which interact with diverse target proteins in a sequence-specific and phosphorylationdependent manner (Bridges and Moorhead, 2005). They have been proved to be involved in many processes of metabolism, hormone signaling introduction, cell division, and responses to abiotic and biotic stress in plants (Chen et al., 2006; Takahashi et al., 2007; Swatek et al., 2011). 14-3-3 proteins participate in $\mathrm{BR}$ signal transduction by regulating the subcellular localization and activity of both BZR1 and BZR2/BES1, which are the key transcription factor of $\mathrm{BR}$ signal transduction (Gampala et al., 2007). Chae et al. (2016) found that 143-3 proteins bound to BRI1, a kind of BR-receptor kinase, and phosphorylated in a BR-dependent manner, demonstrating that 14-3-3 proteins play an important role in the BR signaling of A. thaliana. Therefore, Glyma.06G094400 and Glyma.06G147600 could play an important role in MSNN because they probably would regulate stem growth through BR signaling pathway.

Glyma.19G160800 is annotated as tryptophan synthase alpha chain. Tryptophan synthase is an enzyme that catalyzes the final two steps in the biosynthesis of tryptophan, which could be converted to indole acetic acid (IAA) via the indole acetaldehyde or indole acetonitrile pathway. Glyma.19G161100 is annotated as auxin-responsive protein IAA. In other words, the two genes function in plant growth by IAA indirectly or directly. IAA is well-known for its strong effect on stimulating elongation in isolated stem segments (Yang et al., 1996), which has previously demonstrated that stem elongation strongly responded to exogenous IAA in light-grown pea (Murayama and Ueda, 1973; Yang et al., 1993). Recent research studies further showed that an auxin gradient was involved in cell proliferation in Arabidopsis and rice (Wang et al., 2018), and that auxin could convert to other forms to keep homeostasis to regulate soybean stem growth and development through various pathways (Jiang et al., 2020). Obviously, the two genes have a function in soybean stem growth and might have a certain relationship with MSNN. The four genes are all related with plant hormone signal transduction. It is necessary to verify the function of these genes in the future.

\section{CONCLUSION}

In this study, by combining linkage analysis and GWAS analysis, a total of 119 QTL associated with MSNN were identified in the FW-RIL population. Among them, 24 were simultaneously identified by the two methods. On the basis of the five QTL repeatedly detected in D1 and D2 and the 36 QTL for MSNN response to density, it was implied that a specific molecular mechanism controlled the MSNN response with the increase in plant density. In addition, 109 QTL were newly found, and four candidate genes were predicted to be closely related to MSNN. These genes could be of great value for MAS of soybean breeding. 


\section{DATA AVAILABILITY STATEMENT}

The original contributions presented in the study are included in the article/Supplementary Material, further inquiries can be directed to the corresponding authors.

\section{AUTHOR CONTRIBUTIONS}

HN and W-XL: conceptualization. HZ, MS, RR, TY, HL, $\mathrm{YH}, \mathrm{CL}$, and XS: investigation. HN: resources, data curation, supervision, and funding acquisition. PW: writing-original draft preparation. $\mathrm{BH}$ and $\mathrm{HN}$ : writing-review and editing. W-XL: project administration. All authors have read and agreed to the published version of the manuscript.

\section{FUNDING}

This research was funded by the Hundred-Thousand and Million Project of Heilongjiang Province for Engineering and Technology Science's Soybean Breeding Technology Innovation

\section{REFERENCES}

Akond, M., Liu, S. M., Schoener, L., Anderson, J. A., Kantartzi, S. K., Meksem, K., et al. (2013). A SNP-based genetic linkage map of soybean using the SoyS-NP6K illumina infinium beadchip genotyping array. J. Plant Genome Sci. 1, 80-89. doi: 10.5147/jpgs.2013.0090

Bernard, R. L. (1972). Two genes affecting stem termination in soybeans. Crop Sci. 12, 235-239. doi: 10.2135/cropsci1972.0011183X0012000 20028x

Bridges, D., and Moorhead, G. B. (2005). 14-3-3 proteins: a number of functions for a numbered protein. Sci. STKE 2005:re10. doi: 10.1126/stke.2962005re10

Butrón, A., Santiago, R., Cao, A., Samayoa, L. F., and Malvar, R. A. (2019). QTLs for resistance to Fusarium ear rot in a multiparent advanced generation inter-cross(MAGIC)maize population. Plant Dis. 103, 897-904. doi: 10.1094/PDIS-09-18-1669-RE

Cavanagh, C., Morell, M., Mackay, I., and Powell, W. (2008). From mutations to MAGIC: resources for gene discovery, validation and delivery in crop plants. Curr. Opin. Plant Biol. 11, 215-221. doi: 10.1016/j.pbi.2008. 01.002

Chae, W. B., Park, Y. J., Lee, K. S., Nou, I. S., and Oh, M. H. (2016). Plant receptor kinases bind and phosphorylate 14-3-3 proteins. Genes Genom. 38, 1111-1119. doi: 10.1007/s13258-016-0468-5

Chang, F. G., Guo, C. Y., Sun, F. L., Zhang, J. S., Wang, Z. L., Kong, J. J., et al. (2018). Genome-wide association studies for dynamic plant height and number of nodes on the main stem in summer sowing soybeans. Front. Plant Sci. 9:1184. doi: 10.3389/fpls.2018.01184

Chapman, A., Pantalone, V. R., Ustun, A., Allen, F. L., Landau-Ellis, D., Trigiano, R. N., et al. (2003). Quantitative trait loci for agronomic and seed quality traits in an $\mathrm{F}_{2}$ and $\mathrm{F}_{4: 6}$ soybean population. Euphytica 129, 387-393. doi: 10.1023/A:1022282726117

Chen, F., Li, Q., Sun, L., and He, Z. (2006). The rice 14-3-3 gene family and its involvement in responses to biotic and abiotic stress. DNA Res. 13, 53-63. doi: 10.1093/dnares/dsl001

Chen, Q. S., Zhang, Z. C., Liu, C. Y., Xin, D. W., Qiu, H. M., Shan, D. P., et al. (2007). QTL analysis of major agronomic traits in soybean. Agric. Sci. China 6, 399-405. doi: 10.1016/S1671-2927(07)60062-5

Doyle, J. J., Doyle, J. L., and Brown, A. H. D. (1990). Analysis of a polyploid complex in Glycine with chloroplast and nuclear DNA. Aust. Syst. Bot. 3:125136. doi: $10.1071 /$ SB9900125 and New Cultivar Breeding (2019ZX16B01) to HN, the National Natural Science Foundation of China (32072015), and the Science Foundation of Heilongjiang Province, China (LH2021C029) to W-XL.

\section{ACKNOWLEDGMENTS}

We would like to thank Professor Zhixi Tian (State Key Laboratory of Plant Cell and Chromosome Engineering, Institute of Genetics and Developmental Biology, Chinese Academy of Sciences, Beijing, China) for conducting genotype analysis on the lines of FW-RIL in previous research studies.

\section{SUPPLEMENTARY MATERIAL}

The Supplementary Material for this article can be found online at: https://www.frontiersin.org/articles/10.3389/fpls.2021. 666796/full\#supplementary-material

Egli, D. B. (2013). The relationship between the number of nodes and pods in soybean communities. Crop Sci. 53:1668. doi: 10.2135/cropsci2012. 11.0663

Fang, C., Ma, Y. M., Wu, S. W., Liu, Z., Wang, Z., Yang, R., et al. (2017). Genome-wide association studies dissect the genetic networks underlying agronomical traits in soybean. Genome Biol. 18:161. doi: 10.1186/s13059-0171289-9

Fang, Y. L., Liu, S. L., Dong, Q. Z., Zhang, K. X., Tian, Z. X., Li, X. Y., et al. (2020). Linkage analysis and multi-locus genome-wide association studies identify QTNs controlling soybean plant height. Front. Plant Sci. 11:9. doi: 10.3389/fpls.2020.00009

Gai, J. Y., Wang, Y. J., Wu, X. L., and Chen, S. Y. (2007). A comparative study on segregation analysis and QTL mapping of quantitative traits in plants-with a case in soybean. Front. Agric. China 1, 1-7. doi: 10.1007/s11703-007-0001-3

Gampala, S. S., Kim, T. W., He, J. X., Tang, W. Q., Deng, Z. P., Bai, M. Y., et al. (2007). An essential role for 14-3-3 proteins in brassinosteroid signal transduction in Arabidopsis. Dev. Cell 13, 177-189. doi: 10.1016/j.devcel.2007.06.009

Heuer, S., Hansen, S., Bantin, J., Brettschneider, R., Kranz, E., Lorz, H., et al. (2001). The maize MADS box gene ZmMADS3 affects node number and spikelet development and is co-expressed with ZmMADS1 during flower development, in egg cells, and early embryogenesis. Plant Physiol. 127, 33-45. doi: 10.1104/pp.127.1.33

Hu, Z. B., Zhang, H. R., Kan, G. Z., Ma, D. Y., Zhang, D., Shi, G. X., et al. (2013). Determination of the genetic architecture of seed size and shape via linkage and association analysis in soybean(Glycine max L. Merr.). Genetica 141, 247-254. doi: 10.1007/s10709-013-9723-8

Huang, B. E., George, A. W., Forrest, K. L., Kilian, A., Hayden, M. J., Morell, M. K., et al. (2012). A multi-parent advanced generation inter-cross population for genetic analysis in wheat. Plant Biotechnol. J. 10, 826-839. doi: 10.1111/j.1467-7652.2012.00702.x

Hyten, D. L., Ik-Yong, C., Song, Q., Specht, J. E., Carter, T. E., Shoemaker, R. C., et al. (2010). A high density integrated genetic linkage map of soybean and the development of a 1536 universal soy linkage panel for quantitative trait locus mapping. Crop Sci. 50, 960-968. doi: 10.2135/cropsci2009. 06.0360

Ikeda, T., Saito, H., Matsuda, R., and Sato, S. (1994). Soybean yield and yield components in two planting patterns. J. Agronomy Crop Sci. 173, 73-78. doi: 10.1111/j.1439-037X.1994.tb00540.x 
Jiang, Z. F., Liu, D. D., Wang, T. Q., Liang, X. L., Cui, Y. H., Liu, Z. H., et al. (2020). Concentration difference of auxin involved in stem development in soybean. J. Integr. Agric. 19, 953-964. doi: 10.1016/S2095-3119(19) 62676-6

Jun, T., Freewalt, K., Michel, A. P., and Mian, R. (2014). Identification of novel QTL for leaf traits in soybean. Plant Breed. 133, 61-66. doi: 10.1111/pbr.12107

Kim, K. S., Diers, B. W., Hyten, D. L., Mian, M. A. R., Shannon, J. G., and Nelson, R. L. (2010). Identification of positive yield QTL alleles from exotic soybean germplasm in two bachcross populations. Theor. Appl. Genet. 125, 1353-1369. doi: 10.1007/s00122-012-1944-1

Kover, P. X., Valdar, W., Trakalo, J., Scarcelli, N., Ehrenreich, I. M., Purugganan, M. D., et al. (2009). A multiparent advanced generation inter-cross to finemap quantitative traits in Arabidopsis thaliana. PLoS Genet. 5:e1000551. doi: 10.1371/journal.pgen.1000551

Lee, S., Freewalt, K. R., McHale, L. K., Song, Q. J., Jun, T. H., Michel, A. P., et al. (2015). A high-resolution genetic linkage map of soybean based on 357 recombinant inbred lines genotyped with BARCSoySNP6K. Mol. Breed. 35, 1-7. doi: 10.1007/s11032-015-0209-5

Li, D. M., Sun, M. M., Han, Y. P., Teng, W. L., and Li, W. B. (2010). Identification of QTL underlying soluble pigment content in soybean stems related to resistance to soybean white mold (Sclerotinia sclerotiorum). Euphytica 172, 49-57. doi: 10.1007/s10681-009-0036-Z

Li, X., Wang, P., Zhang, K., Liu, S., Qi, Z., Fang, Y., et al. (2021). Fine mapping QTL and mining genes for protein content in soybean by the combination of linkage and association analysis. Theor. Appl. Genet. 134, 1095-1122. doi: 10.1007/s00122-020-03756-0

Li, X., Xue, H., Zhang, K., Li, W., Fang, Y., Qi, Z., et al. (2019). Mapping QTLs for protein and oil content in soybean by removing the influence of related traits in a four-way recombinant inbred line population. J. Agric. Sci. 157, 659-675. doi: 10.1017/S0021859620000040

Li, X., Zhang, K., Sun, X., Huang, S., Wang, J., Yang, C., et al. (2020). Detection of QTL and QTN and candidate genes for oil content in soybean using a combination of four-way-RIL and germplasm populations. Crop J. 8, 802-811. doi: 10.1016/j.cj.2020.07.004

Liu, R. X., Gong, J. W., and Xiao, X. H., Zhang, Z. (2018). GWAS analysis and QTL identification of fiber quality traits and yield components in upland cotton using enriched high-density SNP markers. Front. Plant Sci. 9:1067. doi: 10.3389/fpls.2018.01067

Liu, S., Xue, H., Zhang, K., Wang, P., Su, D., Li, W., et al. (2019). Mapping QTL affecting the vertical distribution and seed set of soybean [Glycine $\max$ (L.) Merr.] pods. Crop J. 7, 694-706. doi: 10.1016/j.cj.2019. 04.004

Liu, W. X., Kim, M. Y., Van, K., Lee, Y. H., Li, H. L., Liu, X. H., et al. (2011). QTL identification of yield-related traits and their association with flowering and maturity in soybean. J. Crop Sci. Biotechnol. 14, 65-70. doi: 10.1007/s12892-010-0115-7

Moongkanna, J., Nakasathien, S., Novitzky, W., Kwanyuen, P., Sinchaisri, P., and Srinives, P. (2011). SSR markers linking to seed traits and total oil content in soybean. Thai J. Ag. Sci. 44, 233-241.

Murayama, K., and Ueda, K. (1973). Short-term response of Pisum stem segments to indole-3-acetic acid. Plant Cell Physiol. 14, 973-979.

Ott, J., Kamatani, Y., and Lathrop, M. (2011). Family-based designs for genome-wide association studies. Nat. Rev. Genet. 12:465. doi: 10.1038/nrg 2989

Qi, Z., Song, J., Zhang, K., Liu, S., Tian, X., Wang, Y., et al. (2020). Identification of QTNs controlling 100-seed weight in soybean using multilocus genomewide association studies. Front. Genet. 11:689. doi: 10.3389/fgene.2020. 00689

Song, J., Sun, X., Zhang, K., Liu, S., Wang, J., Yang, C., et al. (2020). Identification of QTL and genes for pod number in soybean by linkage analysis and genome-wide association studies. Mol. Breed. 40:60. doi: 10.1007/s11032-020$01140-\mathrm{w}$

Swatek, K. N., Graham, K., Agrawal, G. K., and Thelen, J. J. (2011). The 14-3-3 isoforms chi and epsilon differentially bind client proteins from developing Arabidopsis seed. J. Proteome Res. 10, 4076-4087. doi: 10.1021/pr $200263 \mathrm{~m}$
Takahashi, Y., Kinoshita, T., and Shimazaki, K. (2007). Protein phosphorylation and binding of a 14-3-3 protein in Vicia guard cells in response to ABA. Plant Cell Physiol. 48, 1182-1191. doi: 10.1093/pcp/pcm093

Tamba, C. L., Ni, Y., and Zhang, Y. (2017). Iterative sure independence screening EM-bayesian LASSO algorithm for multi-locus genome-wide association studies. PLoS Comput. Biol. 13:e1005357. doi: 10.1371/journal.pcbi.10 05357

Tanksley, S. D., Ganal, M. W., Prince, J. P., Mc, D. V., Bonierbale, M. W., Broun, P., et al. (1992). High density molecular linkage maps of the tomato and potato genomes. Genetics 132, 1141-1160. doi: 10.1093/genetics/132.4.1141

Tian, X., Zhang, K., Liu, S., Sun, X., Li X, Song, J., et al. (2020). Quantitative trait locus analysis of protein and oil content in response to planting density in soybean (Glycine max [L.] Merri.) seeds based on SNP linkage mapping. Front. Genet. 11:563. doi: 10.3389/fgene.2020.00563

Wang, B., Smith, S. M., and Li, J. (2018). Genetic regulation of shoot architecture. Annu. Rev. Plant Biol. 69, 437-468. doi: 10.1146/annurev-arplant-042817-040422

Wang, P., Sun, X., Zhang, K., Fang, Y., Wang, J., Yang, C., et al. (2021). Mapping QTL/QTN and mining candidate genes for plant height and its response to planting densities in soybean [Glycine max (L.) Merr.] through a FW-RIL population. Mol. Breed. 41:12. doi: 10.1007/s11032-021-01209-0

Wang, S. B., Feng, J. Y., Ren, W. L., Huang, B., Zhou, L., Wen, Y., et al. (2016). Improving power and accuracy of genome-wide association studies via a multi-locus mixed linear model methodology. Sci. Rep. 6:19444. doi: $10.1038 /$ srep 19444

Wang, Z. Y., Seto, H., Fujioka, S., Yoshida, S., and Chory, J. (2001). BRI1 is a critical component of a plasma-membrane receptor for plant steroids. Nature 410, 380-383. doi: 10.1038/35066597

Wen, Y. J., Zhang, H., Ni, Y. L., Huang, B., Zhang, J., Feng, J. Y., et al. (2018). Methodological implementation of mixed linear models in multi-locus genome-wide association studies. Brief. Bioinform. 19, 700-712. doi: 10.1093/bib/bbw145

Yang, T., Davies, P. J., and Reid, J. B. (1996). Genetic dissection of the relative roles of auxin and gibberellin in the regulation of stem elongation in intact light-crown peas. Plant Physiol. 110, 1029-1034. doi: 10.1104/pp.110. 3.1029

Yang, T., Law, D. M., and Davies, P. J. (1993). Magnitude and kinetics of stem elongation induced by exogenous indole-3-acetic acid in intact lightgrown pea seedlings. Plant Physiol. 102, 717-724. doi: 10.1104/pp.102. 3.717

Yao, D., Liu, Z. Z., Zhang, J., Liu, S. Y., Qu, J., Guan, S. Y., et al. (2015). Analysis of quantitative trait loci for main plant traits in soybean. Genet. Mol. Res. 14, 6101-6109. doi: 10.4238/2015.June.8.8

Yu, L. H., Umeda, M., Liu, J. Y., Zhao, N. M., and Uchimiya, H. (1998). A novel MT gene of rice plants in strongly expressed in the node portion of the stem. Gene 206, 29-35. doi: 10.1016/S0378-1119(97) 00577-5

Zhang, J., Feng, J. Y., Ni, Y. L., Wen, Y. J., Niu, Y., Tamba, C. L., et al. (2017). pLARmEB: integration of least angle regression with empirical Bayes for multilocus genome-wide association studies. Heredity 118, 517-524. doi: 10.1038/hdy.2017.8

Zhang, K., Liu, S., Li, W., Liu, S., Li, X., Fang, Y., et al. (2018). Identification of QTNs controlling seed protein content in soybean using multi-locus genomewide association studies. Front. Plant Sci. 9:1690. doi: 10.3389/fpls.2018. 01690

Zhang, S., Meng, L., Wang, J., and Zhang, L. (2017). Background controlled QTL mapping in pure-line genetic populations derived from four-way crosses. Heredity 119, 256-264. doi: 10.1038/hdy.2017.42

Zhang, T. F., Wu, T. T., Wang, L. W., Jiang, B. J., Zhen, C. X., Yuan, S., et al. (2019) A combined linkage and GWAS analysis identifies QTLs linked to soybean seed protein and oil content. Int. J. Mol. Sci. 20:5915. doi: 10.3390/ijms202 35915

Zhang, W. K., Wang, Y. J., Luo, G. Z., Zhang, J. S., He, C. Y., Wu, X. L., et al. (2004). QTL mapping of ten agronomic traits on the soybean (Glycine max L. Merr) genetic map and their association with EST markers. Theor. Appl. Genet. 108, 1131-1139. doi: 10.1007/s00122-003-1527-2 
Zhang, Y. C., Hu, Y., Guan, Z. R., and Li, P. (2020). Combined linkage mapping and association analysis reveals genetic control of maize kernel moisture content. Physiol. Plant. 170, 508-518. doi: 10.1111/ppl.13180

Zhang, Y. W., Tamba, C. L., Wen, Y. J., Li, P., Ren, W. L., Ni, Y. L., et al. (2020). mrMLM v4.0.2: an R platform for multi-locus genomewide association studies. Genomics Proteomics Bioinformatics 18, 481-487. doi: 10.1016/j.gpb.2020.06.006

Zhu, J. (1995). Analysis of conditional genetic effects and variance components in developmetal genetics. Genetics 141, 1633-1639. doi: 10.1093/genetics/141.4.1633

Conflict of Interest: The authors declare that the research was conducted in the absence of any commercial or financial relationships that could be construed as a potential conflict of interest.
Publisher's Note: All claims expressed in this article are solely those of the authors and do not necessarily represent those of their affiliated organizations, or those of the publisher, the editors and the reviewers. Any product that may be evaluated in this article, or claim that may be made by its manufacturer, is not guaranteed or endorsed by the publisher.

Copyright (c) 2021 Li, Wang, Zhao, Sun, Yang, Li, Hou, Liu, Siyal, Raja veesar, Hu and Ning. This is an open-access article distributed under the terms of the Creative Commons Attribution License (CC BY). The use, distribution or reproduction in other forums is permitted, provided the original author(s) and the copyright owner(s) are credited and that the original publication in this journal is cited, in accordance with accepted academic practice. No use, distribution or reproduction is permitted which does not comply with these terms. 